\title{
Quantum-Limited Estimation of Range and Velocity
}

\author{
Zixin Huang $\odot,{ }^{1,2, *}$ Cosmo Lupo $\odot,{ }^{1}$ and Pieter $\mathrm{Kok}^{1}$ \\ ${ }^{1}$ Department of Physics and Astronomy, The University of Sheffield, Sheffield S3 7RH, United Kingdom \\ ${ }^{2}$ Center for Engineered Quantum Systems, Department of Physics and Astronomy, Macquarie University, New \\ South Wales 2109, Australia
}

(Received 21 November 2020; revised 19 April 2021; accepted 21 June 2021; published 6 July 2021)

\begin{abstract}
The energy-time uncertainty relation puts a fundamental limit on the precision of lidars for the estimation of range and velocity. The precision in the estimation of the range (through the time of arrival) and the velocity (through Doppler frequency shifts) of a target are inversely related to each other and are dictated by the bandwidth of the signal. Here, we use the theoretical toolbox of multiparameter quantum metrology to determine the ultimate precision of the simultaneous estimation of range and velocity. We consider the case of a single target as well as a pair of closely separated targets. In the latter case, we focus on the relative position and velocity. We show that the tradeoff between the estimation precision of position and velocity is relaxed for entangled probe states and is completely lifted in the limit of perfect photon time-frequency correlations. In the regime where the two targets are close to each other, the relative position and velocity can be estimated nearly optimally and jointly, even without entanglement, using the measurements determined by the symmetric logarithmic derivatives.
\end{abstract}

DOI: 10.1103/PRXQuantum.2.030303

\section{INTRODUCTION}

Quantum metrology [1,2] and quantum imaging [3] aim at exploiting physical resources such as quantum coherence and entanglement to achieve precision measurements and image resolution beyond those that are allowed by classical physics. A number of applications harness quantum correlations in the energy-time degrees of freedom [4], including lithography [5], quantum-enhanced positioning [6], quantum illumination [7-9], phase estimation [10-12], and ghost imaging [13]. The ability to accurately measure the temporal and spectral properties of light has led to significant developments in chemical spectroscopy [14], ranging [15,16], clock synchronization [17], continuous-variable superdense coding [18], and quantum key distribution $[19,20]$.

In lidars, electromagnetic pulses are sent to interrogate a region of interest and the back-reflected signals are collected and examined. The standard technique to resolve the longitudinal position of the target is based on a measurement of the time of flight associated with a round trip. Furthermore, the radial velocity of the target can be

*zixin.huang@mq.edu.au

Published by the American Physical Society under the terms of the Creative Commons Attribution 4.0 International license. Further distribution of this work must maintain attribution to the author(s) and the published article's title, journal citation, and DOI. deduced by examining the Doppler frequency shift of the returned signals. In this paper, we analyze a model where faint pulses, containing at most one photon, are sent to interrogate a region of space that may contain one or two targets. The photons are prepared in wave packets with central frequency $\omega$. Our results are applicable within the paraxial approximation, in the limiting regime where the background noise is negligible, and the targets can be considered to be pointlike, i.e., their internal structure is unresolved.

If a pulse encounters a single target at distance $x$ that moves with relative (nonrelativistic) radial velocity $v$, then the back-reflected photon (in a lossless scenario) will return after a time delay $\tau$, with its central frequency shifted to $\omega^{\prime}=\omega+\delta v$. The range $x$ and velocity $v$ of the target can thus be estimated as $x \simeq c \tau / 2$, and $v \simeq$ $c(\delta v / \omega) / 2$, where $c$ is the speed of light. If, instead, there are two close targets in the region of interest, with radial position $x_{1}, x_{2}$ and velocity $v_{1}, v_{2}$, a measurement of the time of arrival and the frequency shift allows us to estimate the central position $\left(x_{1}+x_{2}\right) / 2$ and velocity $\left(v_{1}+v_{2}\right) / 2$, as well as the relative position $x_{1}-x_{2}$ and relative velocity $v_{1}-v_{2}$.

The precision in the estimation of the time of arrival and of the signal frequency is $[6,21,22]$

$$
\delta t \simeq \frac{\sigma_{t}}{\sqrt{v n}}, \quad \delta \omega \simeq \frac{\sigma_{\omega}}{\sqrt{\nu n}}
$$


where $\sigma_{\omega}\left(\sigma_{t}\right)$ is the frequency (time) width of the signal, $v$ is the number of pulses used, and $n$ is the number of photons in each pulse. The Arthurs-Kelly relation [23] expresses the fundamental precision limit on the joint estimation of these parameters:

$$
\sigma_{t} \sigma_{\omega} \geqslant 1
$$

Thus for nonentangled photons, lidar systems are subject to a fundamental tradeoff in their ability to resolve the range and velocity of the target.

It is known that uncertainty relations may change in the presence of entanglement [24-26], a phenomenon that could be exploited to boost the precision of quantumlimited range and velocity detection. We use the toolbox of quantum information theory, in particular multiparameter quantum metrology, to assess the ultimate precision of quantum-limited lidars, with or without the assistance of entanglement. We consider the regime of faint pulses with at most one photon each, modeled using a Gaussian envelope. Within this model, we study the problem of jointly estimating the position and velocity of a target, as well as the relative position and velocity of two close targets.

Position and velocity estimation translates into time and frequency estimation, which has been considered before. The estimation of time and frequency shifts following the detection of a single target has previously been studied by Zhuang et al. in the limit of very large entanglement [26]. Here, we consider time and frequency estimation for general probe states with entanglement quantified by a continuous parameter $\kappa \in[0,1)$, where $\kappa=1$ corresponds to perfect time-frequency correlations arising from perfect phase-matching conditions. The estimation of the relative time and frequency for two pulses has been considered by Silberhorn and collaborators [27,28] but they did not consider the use of entanglement and the simultaneous estimation of these parameters.

The structure of the paper is as follows. In Sec. II, we briefly review the tools for multiparameter quantum parameter estimation. In Sec. III, we introduce our model. Section IV presents the ultimate limit in the estimation of position and velocity of a target. In Sec. V, we determine the ultimate limit in the estimation of the relative position and velocity of two targets and an optimal measurement strategy (for the estimation of the relative position) is presented in Sec. VI.

\section{THEORETICAL TOOLBOX}

A quantum parameter estimation routine typically consists of three stages, followed by a classical dataprocessing step. This is shown schematically in Fig. 1(a). First, a quantum system is prepared in a known quantum state. Second, the quantum system is used to probe a target system that we want to investigate. Third, the (a) State preparation

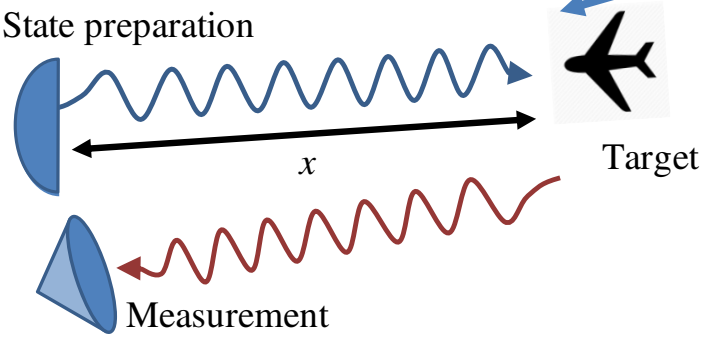

(b)

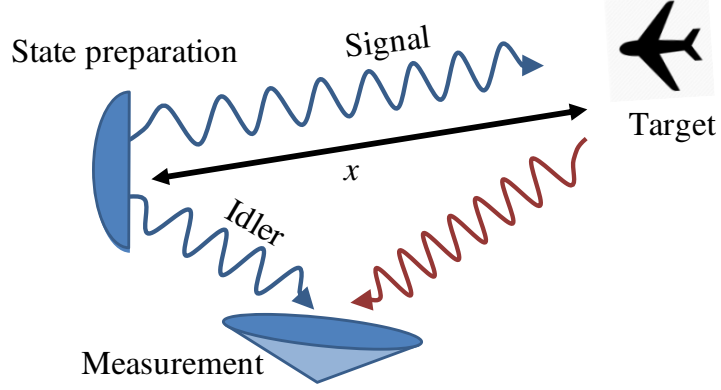

FIG. 1. The use of a lidar for measuring the velocity and range, using (a) separable and (b) entangled states.

probe is measured after interaction with the target. In nonadaptive estimation strategies, the above is repeated $N$ times. Finally, the raw data collected are processed to extract a best estimate for the parameters of interest. An entanglement-assisted strategy refers to the scenario where the probe is initially entangled with an auxiliary system. The latter does not interact with the target but it is jointly measured with the probe. This is shown in Fig. 1(b).

The ultimate precision in the estimation is given by the quantum Cramér-Rao (QCR) bound [29,30]. For the estimation of the parameter $\lambda$ encoded onto a quantum state $\rho_{\lambda}$, this is a lower bound on the variance $\Delta \hat{\lambda}^{2}=\left\langle\hat{\lambda}^{2}\right\rangle-$ $\langle\hat{\lambda}\rangle^{2}$ of any unbiased estimator $\hat{\lambda}$. For unbiased estimators, the QCR bound establishes that

$$
\Delta \hat{\lambda}^{2} \geqslant \frac{1}{N} \frac{1}{J\left(\rho_{\lambda}\right)},
$$

where $N$ is the number of probe systems used and $J$ is the quantum Fisher information (QFI) associated with the global state $\rho_{\lambda}$ of the probes. The latter is defined as

$$
J\left(\rho_{\lambda}\right)=\operatorname{Tr}\left(L_{\lambda}^{2} \rho_{\lambda}\right),
$$

where $L_{\lambda}$ is the symmetric logarithmic derivative (SLD) associated with the parameter $\lambda$ [31]. If the state $\rho_{\lambda}$ lives in a Hilbert space of dimension $d$, consider a set of basis vectors $\left|e_{1}\right\rangle,\left|e_{2}\right\rangle, \ldots,\left|e_{d}\right\rangle$ in which $\rho_{\lambda}$ is diagonal:

$$
\rho_{\lambda}=\sum_{n} p_{n}\left|e_{n}\right\rangle\left\langle e_{n}\right| .
$$


The SLD is then given by

$$
L_{\lambda}=2 \sum_{n, m: p_{n}+p_{m} \neq 0} \frac{\left\langle e_{m}\left|\partial_{\lambda} \rho\right| e_{n}\right\rangle}{p_{n}+p_{m}}\left|e_{m}\right\rangle\left\langle e_{n}\right|,
$$

with $\partial_{\lambda} \rho=\left(\partial \rho_{\lambda} / \partial \lambda\right)$. The QCR bound is asymptotically saturated, that is, there exists a measurement strategy and an unbiased estimator such that Eq. (3) is tight in the limit that $N \rightarrow \infty$ [32]. The SLD directly determines an optimal measurement, which is a projective measurement in the eigenvectors of the SLD operator.

When the quantum state $\rho_{\lambda}$ carries information about multiple parameters, $\lambda=\lambda_{1}, \ldots, \lambda_{K}$, the statistical error in their estimation is expressed by the covariance matrix of the estimators $\hat{\lambda}=\hat{\lambda}_{1}, \ldots, \hat{\lambda}_{K}$,

$$
\operatorname{Cov}[\hat{\lambda}]_{i j}=\left\langle\hat{\lambda}_{i} \hat{\lambda}_{j}\right\rangle-\left\langle\hat{\lambda}_{i}\right\rangle\left\langle\hat{\lambda}_{j}\right\rangle
$$

The multiparameter QCR bound establishes the fundamental lower bound on the covariance matrix of any set of unbiased estimators. This is expressed as a matrix inequality,

$$
\operatorname{Cov}[\hat{\lambda}] \geqslant \frac{1}{N} J(\lambda)^{-1},
$$

where $J(\lambda)$ is the QFI matrix, defined as

$$
J(\lambda)_{i j}=\frac{1}{2} \operatorname{Tr}\left(\rho_{\lambda}\left\{L_{\lambda_{i}}, L_{\lambda_{j}}\right\}\right)
$$

Unlike the single-parameter case, there might not exist a single measurement that allows us to jointly estimate $K>$ 1 parameters simultaneously and optimally. This means that the multiparameter QCR bound is not always achievable. A sufficient condition for the joint and optimal estimation is that the SLD operators commute. A weaker condition, which is necessary and sufficient, is

$$
\operatorname{Tr}\left(\rho_{\lambda}\left[L_{\lambda_{i}}, L_{\lambda_{j}}\right]\right)=0 .
$$

If this condition holds, then there exists a single measurement and a set of $K$ estimators that saturate the multiparameter QCR bound in the limit that $N \rightarrow \infty$ [33-35].

When the condition in Eq. (10) is not met, one can use an alternative bound based on the so-called right logarithmic derivative (RLD). In their analysis, Zhuang et al. have employed this bound [26], the particular form of which has been proved by Fujiwara [36]. While the RLD bound is tighter for this particular problem [37], the RLD operator does not directly relate to a measurement operator. Here, we use the SLD bound, since we know that the SLD translates directly to a measurement operator, and we carefully consider the attainability of the QCR bound.

\section{THE MODEL}

We consider signals with a Gaussian envelope in frequency, which achieves the minimum duration-bandwidth product $\sigma_{t} \sigma_{\omega}=1 / 2$ [38]. By considering Gaussian pulses, we are able to obtain an exact analytical expression for the QFI matrix. We expect that the same qualitative results apply to non-Gaussian pulses under fairly general assumptions.

Consider the field operator $a_{\omega}^{\dagger}$, which creates a photon at frequency $\omega$. To keep the model simple, we do not consider the polarization and spatial degrees of freedom. A state of the quantum electromagnetic field with one photon at frequency $\omega$ is denoted as $|\omega\rangle=a_{\omega}^{\dagger}|\hat{0}\rangle$, where $|\hat{0}\rangle$ is the vacuum. A Gaussian pulse with central frequency $\bar{\omega}_{0}$, central time $\bar{t}_{0}$, and frequency bandwidth $\sigma_{0}$, is described by the state

$$
\left|\psi_{0}\right\rangle=\int d \omega \tilde{\psi}_{0}(\omega)|\omega\rangle
$$

where $|\omega\rangle$ is the single-photon state with frequency $\omega$ and

$$
\tilde{\psi}_{0}(\omega)=\left(\frac{1}{2 \pi \sigma_{0}^{2}}\right)^{\frac{1}{4}} \exp \left[-\frac{\left(\omega-\bar{\omega}_{0}\right)^{2}}{4 \sigma_{0}^{2}}+i \omega \bar{t}_{0}\right] .
$$

Alternatively, we can represent the state in the time domain as

$$
\left|\psi_{0}\right\rangle=\int d t \psi(t)|t\rangle
$$

where

$$
\psi_{0}(t)=\left(\frac{2 \sigma_{0}^{2}}{\pi}\right)^{\frac{1}{4}} \exp \left[-\left(t-\bar{t}_{0}\right)^{2} \sigma_{0}^{2}-i \bar{\omega}_{0}\left(t-\bar{t}_{0}\right)\right]
$$

Here, $|t\rangle=a_{t}^{\dagger}|\hat{0}\rangle$ is the state of a single photon that is detected at time $t$ and $a_{t}^{\dagger}=(2 \pi)^{-1 / 2} \int e^{i \omega t} a_{\omega}^{\dagger}$.

The signal is sent toward a target at distance $x$ that is moving with radial velocity $v$ (we choose $v$ positive when the target is moving away). If the photon in state $|t\rangle$ is backscattered by the target, it is received at time

$$
\tau(t)=t+\frac{2 x}{c(1-\beta)}+\frac{2 \beta\left(t-\bar{t}_{0}\right)}{1-\beta},
$$

where $\beta=v / c$ and $x$ is the position of the target at time $\bar{t}_{0}$. Therefore, the returned photon is described by the state

$$
|\psi\rangle=\int d t \psi_{0}(t)|\tau(t)\rangle=\int d \tau \psi(\tau)|\tau\rangle
$$


where

$$
\begin{aligned}
\psi(\tau)= & \mathcal{N} \exp \left[-\left(\frac{1-\beta}{1+\beta}\left(\tau-t_{0}\right)-\frac{2 x}{c(1+\beta)}\right)^{2} \sigma^{2}\right] \\
& \times \exp \left[-i \omega_{0}\left(\frac{1-\beta}{1+\beta}\left(\tau-t_{0}\right)-\frac{2 x}{c(1+\beta)}\right)\right]
\end{aligned}
$$

$$
=\left(\frac{2 \sigma^{2}}{\pi}\right)^{\frac{1}{4}} \exp \left[-(\tau-\bar{t})^{2} \sigma^{2}-i \bar{\omega}(\tau-\bar{t})\right] .
$$

The latter describes a single-photon state with bandwidth

$$
\sigma=\frac{1-\beta}{1+\beta} \sigma_{0},
$$

and central time and frequency

$$
\begin{gathered}
\bar{t}=\bar{t}_{0}+\frac{2 x}{c(1-\beta)}, \\
\bar{\omega}=\frac{1-\beta}{1+\beta} \bar{\omega}_{0} .
\end{gathered}
$$

The above shows that the information about the target distance $x$ is carried by central time $\bar{t}$ and that the information about the target velocity is carried by all three parameters $\bar{t}, \bar{\omega}$, and $\sigma$. The same relations hold for the case of entanglement-assisted estimation.

For entanglement-assisted sensing, we consider a model of a two-photon state as it is produced by spontaneous parametric down-conversion [24],

$$
\left|\Psi_{0}\right\rangle=\int d \omega \int d \omega_{i} \tilde{\Psi}_{0}\left(\omega, \omega_{i}\right)|\omega\rangle\left|\omega_{i}\right\rangle
$$

where

$$
\begin{aligned}
\tilde{\Psi}_{0}\left(\omega, \omega_{i}\right)= & \tilde{\mathcal{N}}_{0} e^{i\left(\omega+\omega_{i}\right) \bar{t}_{0}} \\
& \times \exp \left[-\frac{\left(\omega-\bar{\omega}_{0}\right)^{2}}{4\left(1-\kappa^{2}\right) \sigma_{0}^{2}}-\frac{\left(\omega_{i}-\bar{\omega}_{i 0}\right)^{2}}{4\left(1-\kappa^{2}\right) \sigma_{i 0}^{2}}\right. \\
& \left.-\frac{\kappa\left(\omega-\bar{\omega}_{0}\right)\left(\omega_{i}-\bar{\omega}_{i 0}\right)}{2\left(1-\kappa^{2}\right) \sigma_{0} \sigma_{i 0}}\right],
\end{aligned}
$$

and $\tilde{\mathcal{N}}_{0}$ is the normalization factor. This two-photon state describes a pair of frequency-entangled photons, with central time $\bar{t}_{0}$, central frequency $\bar{\omega}_{0}, \bar{\omega}_{i 0}$, and bandwidth $\sigma_{0}, \sigma_{i 0}$, for the signal and idler photons respectively. The parameter $\kappa \in[0,1)$ quantifies the amount of entanglement between the signal and idler photon. When $\kappa=0$, the photon pair is separable, whereas in the limit when $\kappa \rightarrow 1$, the photons are perfectly entangled in frequency. Note that the state is nonphysical for $\kappa=1$.

In the time domain, the two-photon state is

$$
\left|\Psi_{0}\right\rangle=\int d t \int d t_{i} \Psi_{0}\left(t, t_{i}\right)|t\rangle\left|t_{i}\right\rangle
$$

with

$$
\begin{aligned}
\Psi_{0}\left(t, t_{i}\right)= & \mathcal{N}_{0} e^{-i \bar{\omega}_{0}\left(t-\bar{t}_{0}\right)-i \bar{\omega}_{i 0}\left(t_{i}-\bar{t}_{0}\right)} \\
& \times \exp \left[-\left(t-\bar{t}_{0}\right)^{2} \sigma_{0}^{2}-\left(t_{i}-\bar{t}_{0}\right)^{2} \sigma_{i 0}^{2}\right. \\
& \left.+2 \kappa\left(t-\bar{t}_{0}\right)\left(t_{i}-\bar{t}_{0}\right) \sigma_{0} \sigma_{i 0}\right] .
\end{aligned}
$$

The signal photon is sent toward the target and the idler is retained, similar to quantum illumination [7-9]. If the signal photon is back-scattered by the target, it will return with a time delay given by Eq. (15). By proceeding as in the single-photon case, we obtain the two-photon state when the returning photon is collected at the receiver:

$$
|\Psi\rangle=\int d t \int d t_{i} \Psi\left(t, t_{i}\right)|t\rangle\left|t_{i}\right\rangle,
$$

where

$$
\begin{aligned}
\Psi\left(t, t_{i}\right)= & \mathcal{N} e^{-i \bar{\omega}(t-\bar{t})-i \bar{\omega}_{i 0}\left(t_{i}-\bar{t}_{0}\right)} \\
& \times \exp \left[-(t-\bar{t})^{2} \sigma^{2}-\left(t_{i}-\bar{t}_{0}\right)^{2} \sigma_{i 0}^{2}\right. \\
& \left.+2 \kappa(t-\bar{t})\left(t_{i}-\bar{t}_{0}\right) \sigma \sigma_{i 0}\right],
\end{aligned}
$$

and the central time $\bar{t}$, central frequency $\bar{\omega}$, and bandwidth $\sigma$ of the signal photon are as in Eqs. (19)-(21). These are the spatiotemporal properties of the back-scattered light, which we use to extract the range and velocity of the targets.

\section{RANGING AND VELOCITY ESTIMATION}

In this section, we present the QFI matrix for the estimation of range and velocity, with and without the assistance of entanglement. As shown in the previous section, in our model the information about range $x$ and velocity $\beta$ (in natural units) of the target is carried by the central time $\bar{t}$, the central frequency $\bar{\omega}$, and the bandwidth $\sigma$ of the returned photon. We first compute the QFI matrix for the estimation of the parameters $\lambda=(\bar{t}, \bar{\omega}, \sigma)$ and then obtain the QFI matrix for the parameters $\boldsymbol{\mu}=(x, \beta)$ that are ultimately of interest. In order to find the ultimate precision of the $\mu$ parameters, we translate their SLDs into the SLDs for the $\lambda$ parameters. We then work with the $\lambda$ parameters throughout the remainder of the paper. 
The SLDs for the $\mu$ parameters are related to the SLDs for the $\lambda$ parameters as follows:

$$
L_{\mu_{j}}=\sum_{k} \frac{\partial \lambda_{k}}{\partial \mu_{j}} L_{\lambda_{k}}
$$

from which we obtain the QFI matrix

$$
J(\boldsymbol{\mu})_{i j}=\sum_{h, k} \frac{\partial \lambda_{h}}{\partial \mu_{i}} \frac{\partial \lambda_{k}}{\partial \mu_{j}} J(\lambda)_{h k} .
$$

The partial derivatives can be evaluated to give

$$
\begin{aligned}
& \frac{\partial \bar{t}}{\partial x}=\frac{2}{c(1-\beta)}, \quad \frac{\partial \bar{t}}{\partial \beta}=\frac{2 x}{c(1-\beta)^{2}}, \\
& \frac{\partial \bar{\omega}}{\partial x}=0, \quad \frac{\partial \bar{\omega}}{\partial \beta}=-\frac{2 \bar{\omega}_{0}}{(1-\beta)^{2}}, \\
& \frac{\partial \sigma}{\partial x}=0, \quad \frac{\partial \sigma}{\partial \beta}=-\frac{2 \sigma}{(1-\beta)^{2}},
\end{aligned}
$$

which yields

$$
\begin{aligned}
L_{x} & =\frac{2}{c(1-\beta)} L_{\bar{t}}, \\
L_{\beta} & =\frac{2}{(1-\beta)^{2}}\left(\frac{x}{c} L_{\bar{t}}-\bar{\omega}_{0} L_{\bar{\omega}}-\sigma L_{\sigma}\right) .
\end{aligned}
$$

Note that these SLDs depend on the central time $\bar{t}$, the central frequency $\bar{\omega}$, and the bandwidth $\sigma$ of the back-scattered photon. Therefore, the SLDs for $x$ and $\beta$ are determined by the SLDs for the $\lambda$ parameters. In the following subsections, we consider this estimation problem with separable and entangled photons.

\section{A. Separable photons}

Firstly, consider using the state in Eq. (14) sent out to the target to estimate the parameters $\lambda=(\bar{t}, \bar{\omega}, \sigma)$. The backscattered photon will have the form given in Eq. (18). This single-photon state lives in an infinite-dimensional Hilbert space. However, as we show in Appendix B, we can compute the QFI matrix by restricting the state to a three-dimensional Hilbert space. We define a suitable system of three basis vectors, $\left|e_{1}\right\rangle=|\psi\rangle$ determined by Eq. (18), $\left|e_{2}\right\rangle$, and $\left|e_{3}\right\rangle$. In this basis, we obtain the following expression for the SLDs:

$$
\begin{aligned}
L_{\bar{t}} & =\left(\begin{array}{ccc}
0 & 2 \sigma & 0 \\
2 \sigma & 0 & 0 \\
0 & 0 & 0
\end{array}\right), L_{\bar{\omega}}=\left(\begin{array}{ccc}
0 & i / \sigma & 0 \\
-i / \sigma & 0 & 0 \\
0 & 0 & 0
\end{array}\right), \\
L_{\sigma} & =\left(\begin{array}{ccc}
0 & 0 & \sqrt{2} / \sigma \\
0 & 0 & 0 \\
\sqrt{2} / \sigma & 0 & 0
\end{array}\right) .
\end{aligned}
$$

From these SLDs, we obtain the QFI matrix

$$
J(\lambda)=\left(\begin{array}{ccc}
4 \sigma^{2} & 0 & 0 \\
0 & 1 / \sigma^{2} & 0 \\
0 & 0 & 2 / \sigma^{2}
\end{array}\right) .
$$

As expected, the QFI of the parameter $\bar{t}$ is inversely proportional to that of $\bar{\omega}$ and it is not possible to make all diagonal elements of $J$ arbitrarily large simultaneously. The tradeoff between time and frequency estimation is expressed by the relation $J(\bar{t}) J(\bar{\omega})=4$.

Since $\rho=|\psi\rangle\left\langle\psi|=| e_{1}\right\rangle\left\langle e_{1}\right|$, the necessary and sufficient condition for joint optimal estimation in Eq. (10) is $\left\langle\psi\left|\left[L_{\lambda_{i}}, L_{\lambda_{j}}\right]\right| \psi\right\rangle=0$. However, we obtain

$$
\left\langle\psi\left|\left[L_{\bar{t}}, L_{\bar{\omega}}\right]\right| \psi\right\rangle=-4 i,
$$

which implies that it is not possible to jointly and optimally estimate the central time and frequency. However, as $\left\langle\psi\left|\left[L_{\bar{t}}, L_{\sigma}\right]\right| \psi\right\rangle=\left\langle\psi\left|\left[L_{\bar{\omega}}, L_{\sigma}\right]\right| \psi\right\rangle=0$, it is possible to estimate jointly the bandwidth and central frequency or the bandwidth and the central time.

After a change of variables, we obtain the SLDs for the position and velocity of the target,

$$
\begin{gathered}
L_{x}=\frac{2}{c(1-\beta)}\left(\begin{array}{ccc}
0 & 2 \sigma & 0 \\
2 \sigma & 0 & 0 \\
0 & 0 & 0
\end{array}\right), \\
L_{\beta}=\frac{2}{(1-\beta)^{2}} \\
0 \\
\left(\begin{array}{ccc}
2 \sigma x / c+i \bar{\omega}_{0} / \sigma & 2 \sigma x / c-i \bar{\omega}_{0} / \sigma & -\sqrt{2} \\
-\sqrt{2} & 0 & 0 \\
& 0 & 0
\end{array}\right) .
\end{gathered}
$$

It follows that

$$
\left\langle\psi\left|\left[L_{x}, L_{\beta}\right]\right| \psi\right\rangle=i \frac{16 \bar{\omega}_{0}}{c(1-\beta)^{3}} .
$$

Thus, the SLDs for $x$ and $\beta$ inherit the incompatibility property of the central time and frequency. This formally shows that it is not possible to jointly estimate $x$ and $\beta$ with separable photons by using the SLD measurement operators and saturating the QCR bound.

\section{B. Entangled photons}

We consider the estimation of the parameters $\lambda=$ $(\bar{t}, \bar{\omega}, \sigma)$ of the two-photon state $|\Psi\rangle$ determined by Eq. (27). As we show in Appendix C, the QFI matrix can be computed within a four-dimensional Hilbert space using 
suitable basis vectors $\left|e_{1}\right\rangle=|\Psi\rangle,\left|e_{2}\right\rangle,\left|e_{3}\right\rangle,\left|e_{4}\right\rangle$. In this basis, the SLDs become

$$
\begin{gathered}
L_{\bar{t}}=\sigma \sqrt{2}\left(\begin{array}{cccc}
0 & \sqrt{1-\kappa} & \sqrt{1+\kappa} & 0 \\
\sqrt{1-\kappa} & 0 & 0 & 0 \\
\sqrt{1+\kappa} & 0 & 0 & 0 \\
0 & 0 & 0 & 0
\end{array}\right), \\
L_{\bar{\omega}}=\frac{1}{\sigma \sqrt{2}}\left(\begin{array}{cccc}
0 & \frac{i}{\sqrt{1-k}} & \frac{i}{\sqrt{1+k}} & 0 \\
-\frac{i}{\sqrt{1-k}} & 0 & 0 & 0 \\
-\frac{i}{\sqrt{1+k}} & 0 & 0 & 0 \\
0 & 0 & 0 & 0
\end{array}\right), \\
L_{\sigma}=\frac{1}{\sigma} \sqrt{\frac{2-\kappa^{2}}{1-\kappa^{2}}}\left(\begin{array}{cccc}
0 & 0 & 0 & 1 \\
0 & 0 & 0 & 0 \\
0 & 0 & 0 & 0 \\
1 & 0 & 0 & 0
\end{array}\right) .
\end{gathered}
$$

For the saturability condition, we obtain

$$
\left\langle\Psi\left|\left[L_{\bar{t}}, L_{\bar{\omega}}\right]\right| \Psi\right\rangle=-4 i,
$$

which is the same as for separable photons. Therefore, the QCR bound cannot be saturated for any $\kappa \in[0,1)$. However, there exists a measurement, not based on the SLDs, which saturates the QCR bound in the limit where $\kappa \rightarrow 1$. This measurement has been constructed by Zhuang et al. [26] and we present it using our approach in Appendix F.

From the SLDs, we obtain the QFI matrix

$$
J(\lambda)=\left(\begin{array}{ccc}
4 \sigma^{2} & 0 & 0 \\
0 & \frac{1}{\sigma^{2}} \frac{1}{1-\kappa^{2}} & 0 \\
0 & 0 & \frac{1}{\sigma^{2}} \frac{2-\kappa^{2}}{1-\kappa^{2}}
\end{array}\right) .
$$

Note that the degree of correlation $1-\kappa^{2}$ appears in the denominator of the QFI for $\bar{\omega}$. This means that the tradeoff in precision between $\bar{t}$ and $\bar{\omega}$ can be lifted by choosing $\kappa$ arbitrarily close to 1 . Moreover, we can make all diagonal elements of $J$ arbitrarily large simultaneously, contrary to the case in Eq. (36). With the assistance of entanglement, we thus obtain

$$
J(\bar{t}) J(\bar{\omega})=\frac{4}{1-\kappa^{2}} .
$$

As previously noted in Ref. [26], this is a violation of the Arthurs-Kelly uncertainty relation [23] for nonentangled photons.

In conclusion, in the limit of $\kappa \simeq 1$, there exists a single measurement (e.g., the measurement discussed in Ref. [26]) such that $\sigma_{\bar{t}}^{2} \simeq 1 / J(\bar{t})$, and $\sigma_{\bar{\omega}}^{2} \simeq 1 / J(\bar{\omega})$, where $\sigma_{\bar{t}}^{2}$
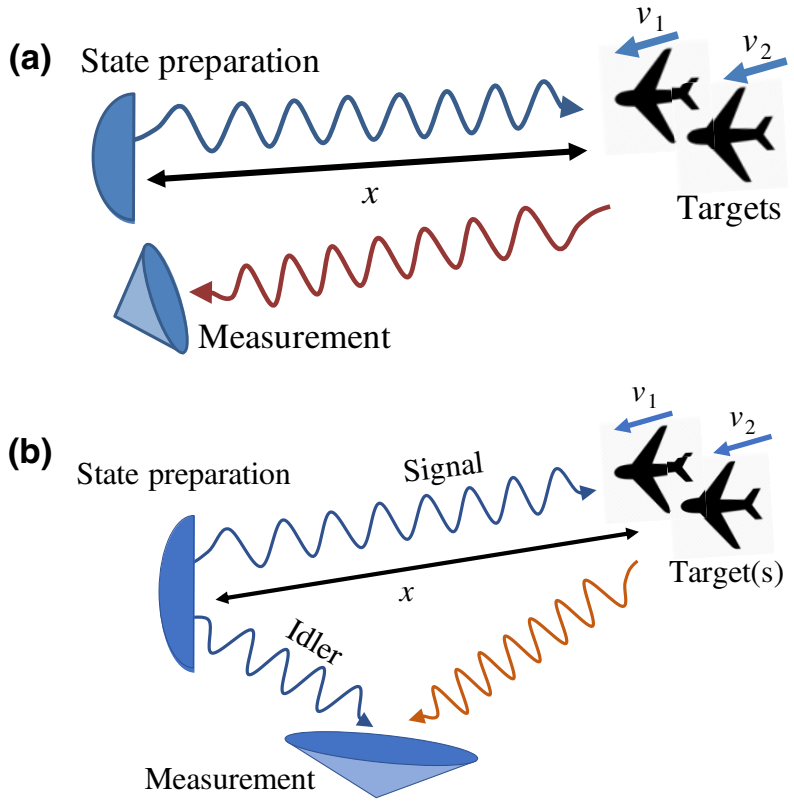

FIG. 2. The use of a lidar for measuring the velocity and range separation using (a) separable and (b) entangled states.

and $\sigma_{\bar{\omega}}^{2}$ are the variances of the estimators of $\bar{t}$ and $\bar{\omega}$. This implies the modified Arthurs-Kelly relation,

$$
\sigma_{\bar{t}} \sigma_{\bar{\omega}} \simeq \frac{\sqrt{1-\kappa^{2}}}{2} \ll \frac{1}{2}
$$

For estimating the individual parameters, the QFI can be achieved by simply heralding the idler photon and then measuring the signal. The QFI for the frequency parameter is inversely proportional to the uncertainty in the frequency of the photon. Conditioning on the measurement outcome of the idler reduces this uncertainty by a factor $\sqrt{1-\kappa^{2}}$ [24]:

$$
\Delta \bar{\omega}=\sqrt{1-\kappa^{2}} \sigma
$$

Therefore, unlike in quantum illumination protocols $[7,8]$, no quantum memory is required to achieve the optimal precision.

\section{TWO TARGETS}

In this section, we focus on the problem of estimating the relative radial position and velocity of two close targets, with either separable [Fig. 2(a)] or entangled [Fig. 2(b)] photons.

In traditional lidar, the ability to measure the separation between two close targets deteriorates if the signals start to overlap. In imaging, the analogous problem, which has been dubbed Rayleigh's curse [39], arises when two objects are closer than the Rayleigh length of the optical 
imaging system. In principle, if the returning signals coherently reflect off the targets, such problems can be bypassed [40]. However, this may not always be the case, especially if the pulses used are in the optical domain. On the other hand, it has been shown that Rayleigh's curse is an artifact of measuring only the intensity of the field and can be avoided by using a suitable coherent detection technique $[39,41]$. The same feature holds for our setting of quantumlimited lidar, as we now show by computing explicitly the QFI matrix for the estimation of the relative distance and velocity of two targets.

We consider a simplified model where the information about the position and velocity of the target is only carried by the central time and central frequency, thus neglecting the bandwidth change. This is justified by the fact that the physics is essentially determined by these two parameters only, as we have shown in detail for the case of a single target.

Assume that target 1 has position $x_{1}$ and velocity $\beta_{1}$ and target 2 has position $x_{2}$ and velocity $\beta_{2}$. If the photon is scattered by target $j$, it will return with central time $\bar{t}_{j}$ and central frequency $\bar{\omega}_{j}$. From Eqs. (20)-(21), we obtain

$$
\begin{aligned}
& \bar{t}_{j}=\bar{t}_{0}+\frac{2 x_{j}}{c\left(1-\beta_{j}\right)}, \\
& \bar{\omega}_{j}=\frac{1-\beta_{j}}{1+\beta_{j}} \bar{\omega}_{0} .
\end{aligned}
$$

Therefore,

$$
\begin{aligned}
\Delta t:=\bar{t}_{1}-\bar{t}_{2} & =\frac{2 x_{1}}{c\left(1-\beta_{1}\right)}-\frac{2 x_{2}}{c\left(1-\beta_{2}\right)} \\
& \simeq \frac{2\left(x_{1}-x_{2}\right)}{c} \\
\Delta \omega:=\bar{\omega}_{1}-\bar{\omega}_{2} & =\frac{1-\beta_{1}}{1+\beta_{1}} \bar{\omega}_{0}-\frac{1-\beta_{2}}{1+\beta_{2}} \bar{\omega}_{0} \\
& \simeq-2\left(\beta_{1}-\beta_{2}\right) \bar{\omega}_{0},
\end{aligned}
$$

where the approximations hold in the nonrelativistic regime $\beta_{1}, \beta_{2} \ll 1$. Putting $\Delta x:=x_{1}-x_{2}$ and $\Delta \beta:=$ $\beta_{1}-\beta_{2}$, we obtain

$$
\frac{\partial \Delta t}{\partial \Delta x}=\frac{2}{c}, \quad \frac{\partial \Delta \omega}{\partial \Delta \beta}=-2 \bar{\omega}_{0} .
$$

This allows us to write the SLDs for the parameters $\Delta x$, $\Delta \beta$ in terms of the SLDs for $\Delta t$ and $\Delta \omega$ :

$$
L_{\Delta x}=\frac{2}{c} L_{\Delta t}, \quad L_{\Delta \beta}=-2 \bar{\omega}_{0} L_{\Delta \omega} .
$$

Below, we first compute the QFI for separable photons and then consider the use of entangled photon pairs.

\section{A. Separable photons}

In this section, we consider an outgoing single-photon state determined by Eq. (14). The back-scattered photon will have the form given in Eq. (18). If the photon returns to the detector, this means that it has been back-scattered by either target 1 or target 2 . As the scattering events are assumed to be incoherent, the returned photon is described by the mixed state

$$
\rho=\frac{1}{2}\left|\psi_{1}\right\rangle\left\langle\psi_{1}\left|+\frac{1}{2}\right| \psi_{2}\right\rangle\left\langle\psi_{2}\right|,
$$

where we assume that the reflectivities of the two objects are approximately equal [42]. We expect that our results also hold for unequal reflectivities [41].

We thus use $\left|\psi_{j}\right\rangle=\int d t \psi_{j}(t)|t\rangle$, for $j=1,2$, with

$$
\psi_{j}(t)=\left(\frac{2 \sigma^{2}}{\pi}\right)^{\frac{1}{4}} \exp \left[-\left(t-\bar{t}_{j}\right)^{2} \sigma^{2}-i \bar{\omega}_{j}\left(t-\bar{t}_{j}\right)\right] .
$$

We define the centroids in time $(T)$ and frequency $(\Omega)$ as (see Fig. 3)

$$
T=\frac{\bar{t}_{1}+\bar{t}_{2}}{2}, \quad \Omega=\frac{\bar{\omega}_{1}+\bar{\omega}_{2}}{2},
$$

The goal of this section is to compute the QFI matrix for the estimation of the parameters $\Delta t$ and $\Delta \omega$.
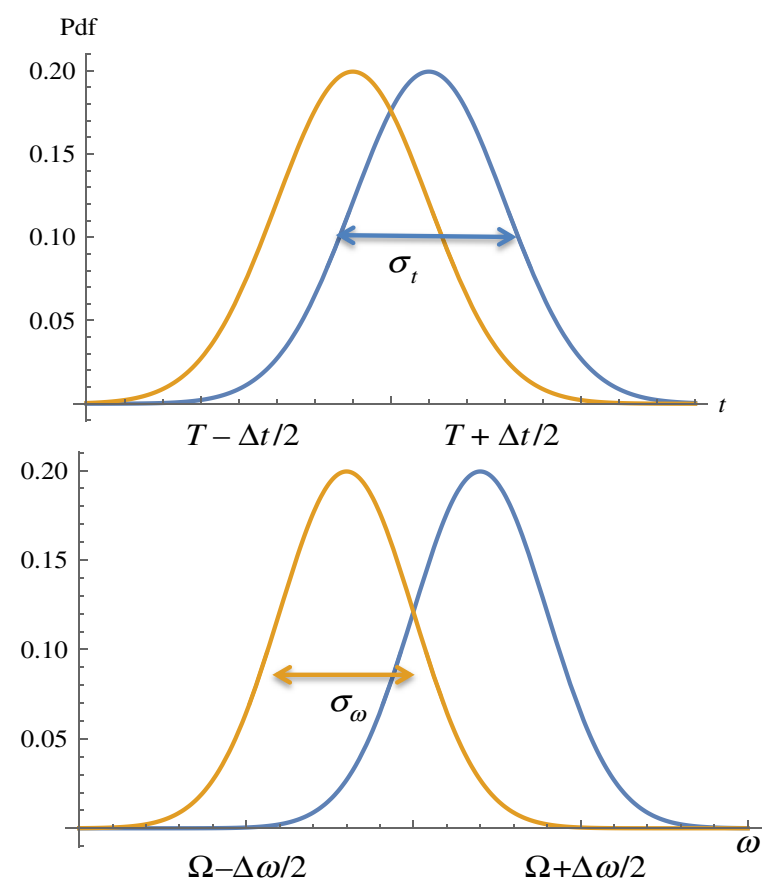

FIG. 3. The time and frequency profiles of arrival of a single photon signal scattering off two objects within the vicinity of each other, where their separation is within the bandwidth of the pulse. 
Following Ref. [39], we obtain an exact expression for the QFI matrix for the estimation of $\Delta t$ and $\Delta \omega$ :

$$
\begin{aligned}
H(\Delta t, \Delta \omega) & =\left(\begin{array}{cc}
\sigma^{2}-\alpha^{-1} \Delta \omega^{2} & \alpha^{-1} \Delta t \Delta \omega \\
\alpha^{-1} \Delta t \Delta \omega & \frac{1}{4 \sigma^{2}}-\alpha^{-1} \Delta t^{2}
\end{array}\right), \\
\alpha & =4\left(e^{\Delta t^{2} \sigma^{2}+\frac{\Delta \omega^{2}}{4 \sigma^{2}}}-1\right)
\end{aligned}
$$

(see Appendix D). The expectation value of the commutator of the SLDs becomes

$$
\begin{aligned}
\operatorname{Tr}\left(\rho\left[L_{\Delta t}, L_{\Delta \omega}\right]\right) & =\frac{4 i}{\alpha}\left(\Delta t^{2} \sigma^{2}+\frac{\Delta \omega^{2}}{4 \sigma^{2}}\right)-i \\
& \simeq-\frac{i}{2}\left(\Delta t^{2} \sigma^{2}+\frac{\Delta \omega^{2}}{4 \sigma^{2}}\right)
\end{aligned}
$$

where the approximation holds, for small values of $\epsilon=$ $\Delta t^{2} \sigma^{2}+\left(\Delta \omega^{2} / 4 \sigma^{2}\right)$, up to correction of order $\epsilon^{2}$. For small values of $\Delta t \sigma$ and $\Delta \omega / \sigma$, this quantity approaches zero and therefore the achievable estimation precision approaches the QCR bound. Note that in this limit, the QFI matrix becomes diagonal. This is in contrast to the single-target problem from the previous section, where the expectation value of the commutator of the SLDs is a constant $-4 i$ [see Eq. (44)]. Some values of $H_{\Delta t^{2}}$ as a function of $\Delta \omega^{2}$ are shown in Fig. 4.

\section{B. Entangled photons}

Next, we consider again the case where the probe photon is entangled with an idler photon; e.g., when both are created in spontaneous parametric down-conversion. If the back-scattering is again incoherent, the two-photon state

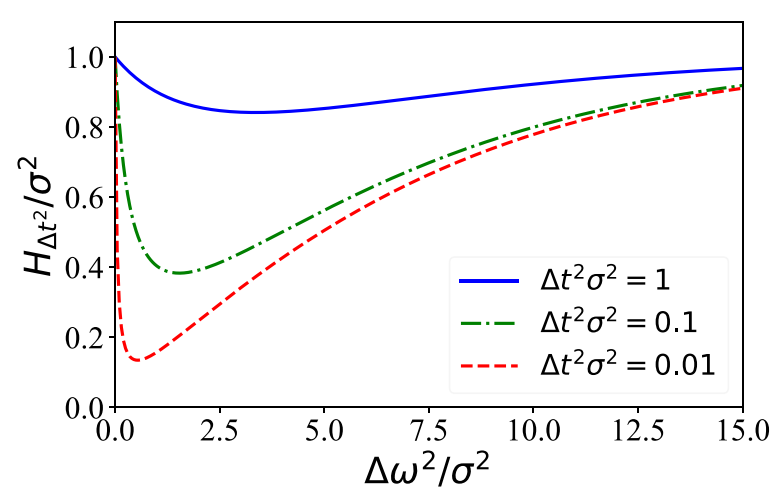

FIG. 4. The QFI matrix component $H_{\Delta t^{2}}$ in Eq. (60) as a function of $\Delta \omega^{2} / \sigma^{2}$, showing $\Delta t^{2} \sigma^{2}=0.01$ (red dashed line), $\Delta t^{2} \sigma^{2}=0.1$ (green dotted-dashed line), and $\Delta t^{2} \sigma^{2}=1$ (blue solid line). becomes

$$
\rho=\frac{1}{2}\left|\Psi_{1}\right\rangle\left\langle\Psi_{1}\left|+\frac{1}{2}\right| \Psi_{2}\right\rangle\left\langle\Psi_{2}\right|,
$$

where $\left|\Psi_{j}\right\rangle=\int d t d t_{i} \Psi_{j}\left(t, t_{i}\right)|t\rangle\left|t_{i}\right\rangle, i$ denotes the idler photon, $j=1,2$, and

$$
\begin{aligned}
\Psi_{j}\left(t, t_{i}\right)= & \mathcal{N} e^{-i \bar{\omega}_{j}\left(t-\bar{t}_{j}\right)-i \bar{\omega}_{i}\left(t_{i}-\bar{t}_{i}\right)} \\
& \times \exp \left[-\left(t-\bar{t}_{j}\right)^{2} \sigma^{2}-\left(t_{i}-\bar{t}_{i}\right)^{2} \sigma_{i}^{2}\right. \\
& \left.+2 \kappa\left(t-\bar{t}_{j}\right)\left(t_{i}-\bar{t}_{i}\right) \sigma \sigma_{i}\right] .
\end{aligned}
$$

This means that although the entangled state lives in a larger Hilbert space, the formal approach used for separable photons can still be applied. As detailed in Appendix E, we obtain the following expression for the QFI matrix:

$$
\begin{aligned}
H_{\mathrm{ent}} & =\left(\begin{array}{cc}
\sigma^{2}-\beta^{-1} \Delta \omega^{2} & \beta^{-1} \Delta \omega \Delta t \\
\beta^{-1} \Delta \omega \Delta t & \frac{1}{4 \sigma^{2}\left(1-\kappa^{2}\right)}-\beta^{-1} \Delta t^{2}
\end{array}\right) \\
\beta & =4\left(e^{\Delta t^{2} \sigma^{2}+\frac{\Delta \omega^{2}}{4\left(1-\kappa^{2}\right) \sigma^{2}}}-1\right) .
\end{aligned}
$$

Here, we see a similar quantitative change in the QFI matrix compared to single-target detection: the term $1-$ $\kappa^{2}$ appears as a multiplicative factor in front of the bandwidth $\sigma_{s}$, which effectively reduces the frequency uncertainty of the probe photon. Provided that $\kappa \neq 0$, the components of the QFI matrix are always larger than in the unentangled case. The expectation value of the commutator of the SLDs becomes

$$
\begin{aligned}
\operatorname{Tr}\left(\rho\left[L_{\Delta t}, L_{\Delta \omega}\right]\right) & =\frac{4 i}{\beta}\left(\Delta t^{2} \sigma^{2}+\frac{\Delta \omega^{2}}{4\left(1-\kappa^{2}\right) \sigma^{2}}\right)-i \\
& \simeq-\frac{i}{2}\left(\Delta t^{2} \sigma^{2}+\frac{\Delta \omega^{2}}{4\left(1-\kappa^{2}\right) \sigma^{2}}\right),
\end{aligned}
$$

which approaches zero for small values of $\Delta t \sigma$ and $\Delta \omega / \sigma$.

In conclusion, we find that if the targets are sufficiently close in both position and velocity, the SLD measurements become compatible up to a small eigenbasis mismatch. In this limit, the two parameters become jointly measurable and the QCR bound can be saturated.

\section{OPTIMAL TIME-DIFFERENCE ESTIMATION WITH LINEAR OPTICS}

The QFI provides us with an upper bound to the ultimate precision but does not always provide the optimal physical measurement. Consider a quantum state $\rho(\lambda)$, which carries information about the parameter $\lambda$. When a given 


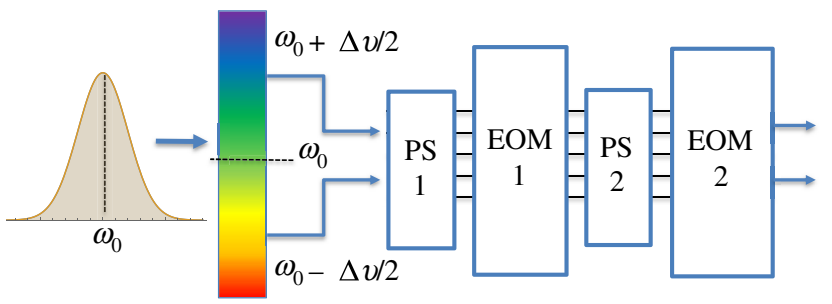

FIG. 5. A linear measurement that achieves the QFI for the parameter $\Delta t$, for the special case where $\Delta \omega=0$, and $\kappa=0$. It involves separating the signal into different frequency modes, then selecting the frequencies equidistant from either side of the central frequency, followed by a frequency Hadamard gate. The schematic for the Hadamard gate consists of two phase shifters (PSs) and two electro-optic modulators (EOMs). Photon counting is then performed at the output.

measurement $M$ is applied to $\rho(\lambda)$, it yields outcomes $\left\{m_{y}\right\}_{y=1, \ldots, Y}$ with probabilities $\left\{p_{y}\right\}_{y=1, \ldots, Y}$. The classical Fisher information (CFI) associated with this measurement is [43]

$$
I(\lambda)=\sum_{y} p_{y}\left(\frac{\partial \log p_{y}}{\partial \lambda}\right)^{2} .
$$

The (classical) Cramér-Rao bound expresses the relation between the CFI and the variance of any unbiased estimator $\hat{\lambda}, \Delta \hat{\lambda} \geq(1 / N) I(\lambda)^{-1}$. An optimal measurement is such that the CFI is equal to the QFI.

Here, we consider the two-target problem and provide an optimal measurement for the estimation of $\Delta t$ when $\Delta \omega=0$, i.e., the two targets are moving at the same velocity. In particular, we focus on the case of separable photons, described by the state in Eq. (57). In this setup, an optimal measurement has been presented in Ref. [27] using a quantum pulse gate. Unlike the quantum pulse gate, which is based on up-conversion, here we propose an approach that requires no optical nonlinearity.

A schematic for an optimal linear measurement is depicted in Fig. 5. The measurement consists of first sending the signal through a diffraction grating, which separates the frequencies within the pulse. Then, one takes the frequencies on either side of the centroid that are equidistant and interfere them through a frequency Hadamard gate. Finally, photon counting is performed at the output.

We now derive the classical Fisher information associated with this particular measurement. Upon the return of the signal from target 1 or 2 , the annihilation operators can be written as

$$
\begin{aligned}
& \hat{a}_{1}(t)=\int d \omega \hat{a}(\omega) e^{-i \omega t_{1}+i \phi_{k 0}}, \\
& \hat{a}_{2}(t)=\int d \omega \hat{a}(\omega) e^{-i \omega t_{2}+i \phi_{k 0}} .
\end{aligned}
$$

Here, we choose $\phi_{k 0}=0$ without loss of generality. Now, we select the frequencies at either side of the central frequency $\bar{\omega}$ separated by $\Delta v: v_{1}=\bar{\omega}+\Delta v / 2$ and $v_{2}=\bar{\omega}-$ $\Delta v / 2$. For these two frequencies, the density matrix of the state upon return can be written as

$$
\begin{aligned}
\rho & =\frac{1}{2}\left(\left|f_{1}\right\rangle\left\langle f_{1}|+| f_{2}\right\rangle\left\langle f_{2}\right|\right), \\
\left|f_{1}\right\rangle & =\frac{1}{\sqrt{2}}\left(\left|v_{1}\right\rangle+e^{i\left(v_{2}-v_{1}\right) t_{1}}\left|v_{2}\right\rangle\right), \\
\left|f_{2}\right\rangle & =\frac{1}{\sqrt{2}}\left(\left|v_{1}\right\rangle+e^{i\left(v_{2}-v_{1}\right) t_{2}}\left|v_{2}\right\rangle\right) .
\end{aligned}
$$

The experimenter implements a frequency Hadamard gate on the states $\left|v_{1}\right\rangle$ and $\left|\nu_{2}\right\rangle$, which is achievable using two phase shifters and electro-optical modulators, i.e., without employing nonlinear optics. Note that no postselection is required for such a Hadamard gate and that it has been experimentally demonstrated in Ref. [44] with nearly unit success probability and fidelity. The Hadamard gate is given by

$$
H=\frac{1}{\sqrt{2}}\left(\begin{array}{cc}
1 & 1 \\
1 & -1
\end{array}\right) .
$$

The state then becomes

$$
\rho^{\prime}=\left(\begin{array}{cc}
p_{1} & b \\
b^{*} & p_{2}
\end{array}\right)
$$

where $b=(1 / 2) \sin \left[t_{2}\left(v_{1}-v_{2}\right)\right]-(i / 2) \sin \left[t_{1}\left(v_{1}-v_{2}\right)\right]$. The diagonal terms give the probabilities of the two measurement outcomes at the output of the Hadamard gate,

$$
\begin{aligned}
& p_{1}=\frac{1}{4}\left\{2+\cos \left[\left(v_{2}-v_{1}\right) t_{1}\right]+\cos \left[\left(v_{2}-v_{1}\right) t_{2}\right]\right\}, \\
& p_{2}=\frac{1}{4}\left\{2-\cos \left[\left(v_{2}-v_{1}\right) t_{1}\right]-\cos \left[\left(v_{2}-v_{1}\right) t_{2}\right]\right\} .
\end{aligned}
$$

The Fisher information of the parameter $\Delta t=t_{1}-t_{2}$ for Eq. (78) is

$$
I(\Delta t)=\left(v_{1}-v_{2}\right)^{2} / 4 .
$$

The above calculation is for a single pair of frequencies. To calculate the overall Fisher information, we average Eq. (80) over the frequency distribution of the signal state. The returning state has a probability density distribution (PDF)

$$
p_{\Omega}(\omega-\bar{\omega})=\left(\frac{1}{2 \pi \sigma^{2}}\right)^{\frac{1}{2}} \exp \left[-\frac{(\omega-\bar{\omega})^{2}}{2 \sigma^{2}}\right],
$$

and the PDF of $\left|v_{1}-v_{2}\right|$ is equal to $p_{\Omega}\left[\left(v_{1}-v_{2}\right) / 2\right]$. Using Eq. (70), the expression for the CFI is

$$
I(\Delta t)=\int_{0}^{\infty} p_{\Omega}\left[\left(v_{1}-v_{2}\right) / 2\right] \frac{\left|v_{1}-v_{2}\right|^{2}}{4}=\sigma^{2} .
$$


Equation (82) is equal to the corresponding element of the QFI in Eq. (60) and thus the measurement is optimal.

Intuitively, the frequency bandwidth of the state plays the same role as the numerical aperture (i.e., the size of the lens) in classical optical imaging. For optical imaging in the paraxial regime, the source distribution is Fourier transformed into its spatial-frequency components. The larger the numerical aperture, the more information one can collect on the spatial distribution of the source. Likewise, if the goal is to collect timing information, the larger the frequency bandwidth, the more accurately one can locate the pulses in the time domain. In both cases, the use of a phasesensitive measurement instead of the intensity profile can avoid Rayleigh's curse.

\section{CONCLUSIONS}

In this paper, we assess the ultimate precision of a lidar using the theoretical toolbox of multiparameter quantum metrology, within the paraxial approximation, in the regime where the targets can be considered pointlike and the background noise can be ignored. We consider both the case of a single target as well as a pair of close targets. In the latter case, we focus on the problem of estimating the relative position and velocity.

Our theory shows that the tradeoff between the estimation of time and frequency can be weakened when the signal photon is entangled and jointly measured with an idler photon. In other words, the bandwidth-duration product is no longer lower bounded from below by $1 / 2$. The more the photon pair is entangled, the more the tradeoff is weakened, and it can be completely removed in the limit of perfect time-frequency correlation. In that case, the QCR bound is attainable. Our results are consistent with what has previously been presented by Zhuang et al. [26] and they elucidate the subtle issues surrounding the estimation of time and frequency.

For the estimation of the relative distance and velocity of two targets, one expects that the precision deteriorates when the two targets are close enough, such that there is a substantial overlap between the two back-scattered signals. This is the lidar analogy of the so-called Rayleigh curse, which is observed in classical optical imaging based on direct photodetection [39]. In previous work, Silberhorn and collaborators have shown that a coherent detection technique allows us to lift Rayleigh's curse and measure the difference in the time of arrival with constant precision, independent of the signal overlap [27,28]. The same holds for the estimation of time or frequency difference.

Here, we consider the joint estimation of both the difference in time of arrival (i.e., the relative position of two targets) and in frequency (i.e., the relative velocity of two targets). In analogy with the case of a single target, we find that there exists a tradeoff between timeand frequency-difference estimation and that this tradeoff can be weakened if entangled photon pairs are employed. However, in contrast to the single-target case and classical intuition, we show that these parameters can be simultaneously estimated, even without entanglement, in the regime where the two signals have a substantial overlap, that is, when the frequency difference is much smaller than the bandwidth and the relative time of arrival is much smaller than the signal duration. Our results will be important for the realistic implementation of superresolution lidar systems with finite entanglement in the probe beam.

\section{ACKNOWLEDGMENTS}

We thank Lorenzo Maccone, Ilaria Gianani, Quntao Zhuang, Zheshen Zhang, Jeffrey H. Shapiro, Rafal Demkowicz-Dobrzanski, and Francesco Albarelli for insightful comments and discussions. This work was supported by the EPSRC Quantum Communications Hub, Grant No. EP/M013472/1.

\section{APPENDIX A: WAVE FUNCTION FOR THE BACK-SCATTERED PHOTON}

Consider a light pulse that leaves the detector station at time $t$. At time $\bar{t}_{0}$, the target is at distance $x$ from the detector and moves with radial velocity $v$. This implies that at time $t$, its position is $x+v\left(t-\bar{t}_{0}\right)$. The light pulse will thus meet the target after a time $\Delta t$ such that $c \Delta t=$ $x+v\left(t-\bar{t}_{0}\right)+v \Delta t$. Therefore,

$$
\Delta t=\frac{x+v\left(t-\bar{t}_{0}\right)}{c-v} .
$$

Hence the total time for the back-scattered signal to come back to the detector station is $2 \Delta t$. In conclusion, a signal that is sent at time $t$ will return at time

$$
\tau(t)=t+2 \frac{x+v\left(t-\bar{t}_{0}\right)}{c-v} .
$$

This result is used in Eq. (15) to obtain the wave function of the back-scattered signal in Eq. (16). The latter can be also obtained in the frequency domain. A signal at frequency $\omega$, when back-scattered by the target moving with velocity $v$, has its frequency shifted to $\omega^{\prime}=$ $(c-v / c+v) \omega$. Using the central time $\bar{t}_{0}$ as a reference, the back-scattered pulse also acquires a phase shift equal to $\exp \left\{-i \omega \bar{t}_{0}+i \omega^{\prime}\left[\bar{t}_{0}+2(x / c-v)\right]\right\}$. Therefore, starting from the input state of Eq. (12),

$$
\left|\psi_{0}\right\rangle=\int d \omega \tilde{\psi}_{0}(\omega)|\omega\rangle,
$$

where

$$
\tilde{\psi}_{0}(\omega)=\left(\frac{1}{2 \pi \sigma_{0}^{2}}\right)^{\frac{1}{4}} \exp \left[-\frac{\left(\omega-\bar{\omega}_{0}\right)^{2}}{4 \sigma_{0}^{2}}+i \omega \bar{t}_{0}\right],
$$


this is mapped into

$$
\begin{aligned}
& \int d \omega \tilde{\psi}_{0}(\omega)|\omega\rangle \rightarrow \int d \omega \tilde{\psi}_{0}(\omega) \\
& \times \exp \left[-i \omega \bar{t}_{0}+i \omega^{\prime}\left(\bar{t}_{0}+2 \frac{x}{c-v}\right)\right]\left|\omega^{\prime}\right\rangle \\
& =\frac{c+v}{c-v} \int d \omega^{\prime} \tilde{\psi}_{0}\left(\frac{c+v}{c-v} \omega^{\prime}\right) \\
& \times \exp \left[-i \omega \bar{t}_{0}+i \omega^{\prime}\left(\bar{t}_{0}+2 \frac{x}{c-v}\right)\right]\left|\omega^{\prime}\right\rangle \\
& =\int d \omega\left(\frac{1}{2 \pi \sigma^{2}}\right)^{\frac{1}{4}} \exp \left[-\frac{(\omega-\bar{\omega})^{2}}{4 \sigma^{2}}+i \omega \bar{t}\right]
\end{aligned}
$$

where

$$
\begin{aligned}
\sigma & =\frac{1-\beta}{1+\beta} \sigma_{0}, \\
\bar{t} & =\bar{t}_{0}+\frac{2 x}{c(1-\beta)}, \\
\bar{\omega} & =\frac{1-\beta}{1+\beta} \bar{\omega}_{0} .
\end{aligned}
$$

It is easy to check that the function in Eq. (A7) is the Fourier transform of that in Eq. (16).

\section{APPENDIX B: RANGING AND VELOCITY ESTIMATION WITH SEPARABLE PHOTONS}

For the unentangled case, we consider the state $|\psi\rangle=$ $\int_{-\infty}^{\infty} d t \psi(t)|t\rangle$, with

$$
\psi(t)=\left(\frac{2 \sigma^{2}}{\pi}\right)^{1 / 4} e^{-(t-\bar{t})^{2} \sigma^{2}} e^{-i \bar{\omega}(t-\bar{t})}
$$

We define the vectors $\left|\phi_{k}\right\rangle=\int_{-\infty}^{\infty} d t \phi_{k}(t)|t\rangle$, for $k=$ $1,2,3,4$, where

$$
\begin{gathered}
\phi_{1}(t):=\psi(t), \\
\phi_{2}(t):=\partial_{\bar{t}} \psi(t)=\left[2(t-\bar{t}) \sigma^{2}+i \bar{\omega}\right] \psi(t), \\
\phi_{3}(t)=\partial_{\bar{\omega}} \psi(t)=-i(t-\bar{t}) \psi(t), \\
\phi_{4}(t)=\partial_{\sigma} \psi(t)=\left[\frac{1}{2 \sigma}-2(t-\bar{t})^{2} \sigma\right] \psi(t) .
\end{gathered}
$$

The local dynamics of the state $|\psi\rangle$, for small variations of the parameters $\bar{t}, \bar{\omega}$, and $\sigma$, are confined within the Hilbert space generated by these four vectors. It is easy to check that the above vectors span a three-dimensional Hilbert space. An orthonormal basis for this space is $\left|e_{j}\right\rangle=$ $\int_{-\infty}^{\infty} d t e_{j}(t)|t\rangle$, for $j=1,2,3$, where

$$
\begin{aligned}
& e_{1}(t)=\psi(t), \\
& e_{2}(t)=2 \sigma(t-\bar{t}) \psi(t), \\
& e_{3}(t)=\frac{1-4(t-\bar{t})^{2} \sigma^{2}}{\sqrt{2}} \psi(t) .
\end{aligned}
$$

In this basis, we obtain the following expression for the SLDs:

$$
\begin{aligned}
L_{\bar{t}} & =2 \sigma\left(\begin{array}{lll}
0 & 1 & 0 \\
1 & 0 & 0 \\
0 & 0 & 0
\end{array}\right), \\
L_{\bar{\omega}} & =\frac{1}{\sigma}\left(\begin{array}{ccc}
0 & i & 0 \\
-i & 0 & 0 \\
0 & 0 & 0
\end{array}\right), \\
L_{\sigma} & =\frac{\sqrt{2}}{\sigma}\left(\begin{array}{lll}
0 & 0 & 1 \\
0 & 0 & 0 \\
1 & 0 & 0
\end{array}\right) .
\end{aligned}
$$

Therefore, the QFI matrix is

$$
H(\bar{t}, \bar{\omega}, \sigma)=\left(\begin{array}{ccc}
4 \sigma^{2} & 0 & 0 \\
0 & 1 / \sigma^{2} & 0 \\
0 & 0 & 2 / \sigma^{2}
\end{array}\right)
$$

We can then obtain the SLDs for the estimation of the position $x$ and velocity $\beta$ of a moving target:

$$
L_{x}=\frac{2}{c(1-\beta)}\left(\begin{array}{ccc}
0 & 2 \sigma & 0 \\
2 \sigma & 0 & 0 \\
0 & 0 & 0
\end{array}\right) \text {, }
$$

$$
L_{\beta}=\frac{2}{(1-\beta)^{2}}\left(\begin{array}{ccc}
0 & 2 \sigma x / c+i \bar{\omega}_{0} / \sigma & \sqrt{2} \\
2 \sigma x / c-i \bar{\omega}_{0} / \sigma & 0 & 0 \\
\sqrt{2} & 0 & 0
\end{array}\right) \text {, }
$$

and

$$
H(x, \beta)=\frac{4}{(1-\beta)^{2}}\left(\begin{array}{cc}
\frac{4 \sigma^{2}}{c^{2}} & \frac{4 x \sigma^{2}}{c^{2}(1-\beta)} \\
\frac{4 x \sigma^{2}}{c^{2}(1-\beta)} & \frac{4 x^{2} \sigma^{4}+c^{2}\left(2 \sigma^{2}+\bar{\omega}_{0}^{2}\right)}{c^{2} \sigma^{2}(1-\beta)^{2}}
\end{array}\right) .
$$




\section{APPENDIX C: RANGING AND VELOCITY ESTIMATION USING ENTANGLED PHOTONS}

In the time domain, the two-photon state reads

$$
|\Psi\rangle=\int d t \int d t_{i} \Phi\left(t, t_{i}\right)|t\rangle\left|t_{i}\right\rangle,
$$

with

$$
\begin{aligned}
\Psi\left(t, t_{i}\right) & =e^{-i \bar{\omega}(t-\bar{t})-i \bar{\omega}_{i}\left(t_{i}-\bar{t}_{i}\right)}\left(1-\kappa^{2}\right)^{1 / 4} \sqrt{\frac{2 \sigma \sigma_{i}}{\pi}} \\
& \exp \left[-(t-\bar{t})^{2} \sigma^{2}-\left(t_{i}-\bar{t}_{i}\right)^{2} \sigma_{i}^{2}+2 \kappa(t-\bar{t})\left(t_{i}-\bar{t}_{i}\right) \sigma \sigma_{i}\right] .
\end{aligned}
$$

Consider the following vectors:

$$
\begin{aligned}
& \Phi_{1}\left(t, t_{i}\right):=\Psi\left(t, t_{i}\right), \\
& \Phi_{2}\left(t, t_{i}\right):=\partial_{\bar{t}} \Psi\left(t, t_{i}\right)=\left[i \bar{\omega}+2(t-\bar{t}) \sigma^{2}-2 \kappa\left(t_{i}-\bar{t}_{i}\right) \sigma \sigma_{i}\right] \Psi\left(t, t_{i}\right), \\
& \Phi_{3}\left(t, t_{i}\right):=\partial_{\bar{\omega}} \Psi\left(t, t_{i}\right)=-i(t-\bar{t}) \Psi\left(t, t_{i}\right), \\
& \Phi_{4}\left(t, t_{i}\right):=\partial_{\sigma} \Psi\left(t, t_{i}\right)=\left[\frac{1}{2 \sigma}-2(t-\bar{t})^{2} \sigma+2 \kappa(t-\bar{t})\left(t_{i}-\bar{t}_{i}\right) \sigma_{i}\right] \Psi\left(t, t_{i}\right) .
\end{aligned}
$$

These vectors generate a four-dimensional Hilbert space. An orthonormal basis for this space is $\left|e_{j}\right\rangle=$ $\int_{-\infty}^{\infty} d t \int_{-\infty}^{\infty} d t_{i} e_{j}\left(t, t_{i}\right)|t\rangle\left|t_{i}\right\rangle$, for $j=1,2,3,4$, where

$$
\begin{aligned}
& e_{1}\left(t, t_{i}\right)=\Psi\left(t, t_{i}\right), \\
& e_{2}\left(t, t_{i}\right)=\sqrt{2(1-\kappa)}\left[\sigma(t-\bar{t})+\sigma_{i}\left(t_{i}-\bar{t}_{i}\right)\right] \Psi\left(t, t_{i}\right), \\
& e_{3}\left(t, t_{i}\right)=\sqrt{2(1+\kappa)}\left[\sigma(t-\bar{t})-\sigma_{i}\left(t_{i}-\bar{t}_{i}\right)\right] \Psi\left(t, t_{i}\right), \\
& e_{4}\left(t, t_{i}\right)=2 \sigma \sqrt{\frac{1-\kappa^{2}}{2-\kappa^{2}}}\left[\frac{1}{2 \sigma}-2\left(t-\bar{t}^{2} \sigma+2 \kappa(t-\bar{t})\left(t_{i}-\bar{t}_{i}\right) \sigma_{i}\right] \Psi\left(t, t_{i}\right) .\right.
\end{aligned}
$$

We then obtain the following expressions for the SLDs:

$$
\begin{aligned}
L_{\bar{t}} & =\sigma \sqrt{2}\left(\begin{array}{cccc}
0 & \sqrt{1-\kappa} & \sqrt{1+\kappa} & 0 \\
\sqrt{1-\kappa} & 0 & 0 & 0 \\
\sqrt{1+\kappa} & 0 & 0 & 0 \\
0 & 0 & 0 & 0
\end{array}\right), \\
L_{\bar{\omega}} & =\frac{1}{\sigma \sqrt{2}}\left(\begin{array}{cccc}
0 & \frac{i}{\sqrt{1-k}} & \frac{i}{\sqrt{1+k}} & 0 \\
-\frac{i}{\sqrt{1-k}} & 0 & 0 & 0 \\
-\frac{i}{\sqrt{1+k}} & 0 & 0 & 0 \\
0 & 0 & 0 & 0
\end{array}\right) \\
L_{\sigma} & =\frac{1}{\sigma} \sqrt{\frac{2-\kappa^{2}}{1-\kappa^{2}}}\left(\begin{array}{cccc}
0 & 0 & 0 & 1 \\
0 & 0 & 0 & 0 \\
0 & 0 & 0 & 0 \\
1 & 0 & 0 & 0
\end{array}\right) .
\end{aligned}
$$

These in turn yield the QFI matrix:

$$
H(\bar{t}, \bar{\omega}, \sigma)=\left(\begin{array}{ccc}
4 \sigma^{2} & 0 & 0 \\
0 & \frac{1}{\sigma^{2}} \frac{1}{1-\kappa^{2}} & 0 \\
0 & 0 & \frac{1}{\sigma^{2}} \frac{2-\kappa^{2}}{1-\kappa^{2}}
\end{array}\right)
$$




\section{APPENDIX D: RELATIVE RANGE AND VELOCITY ESTIMATION WITH SEPARABLE PHOTONS}

For the unentangled case, we consider the state

$$
\rho=\frac{1}{2}\left(\left|\psi_{1}\right\rangle\left\langle\psi_{1}|+| \psi_{2}\right\rangle\left\langle\psi_{2}\right|\right),
$$

where

$$
\left|\psi_{1}\right\rangle=\int_{-\infty}^{\infty} d t \psi_{1}(t)|t\rangle, \quad\left|\psi_{2}\right\rangle=\int_{-\infty}^{\infty} d t \psi_{2}(t)|t\rangle
$$

and

$$
\begin{aligned}
& \psi_{1}(t)=\left(\frac{2 \sigma^{2}}{\pi}\right)^{1 / 4} e^{-\left(t-\bar{t}_{1}\right)^{2} \sigma^{2}} e^{-i \bar{\omega}_{1}\left(t-\bar{t}_{1}\right)} \\
& \psi_{2}(t)=\left(\frac{2 \sigma^{2}}{\pi}\right)^{1 / 4} e^{-\left(t-\bar{t}_{2}\right)^{2} \sigma^{2}} e^{-i \bar{\omega}_{2}\left(t-\bar{t}_{2}\right)}
\end{aligned}
$$

We write the time and frequency in terms of their centroids and separations,

$$
\begin{array}{ll}
\bar{t}_{1}=\Sigma t+\Delta t / 2, & \bar{\omega}_{1}=\Sigma \omega+\Delta \omega / 2 \\
\bar{t}_{2}=\Sigma t-\Delta t / 2, & \bar{\omega}_{2}=\Sigma \omega-\Delta \omega / 2 .
\end{array}
$$

An orthonormal basis for this space is

$$
\begin{aligned}
& \left|e_{1}\right\rangle=\frac{1}{\sqrt{2(1+|\delta|)}}\left(\left|\psi_{1}\right\rangle+e^{i \Delta t \Sigma \omega}\left|\psi_{2}\right\rangle\right), \\
& \left|e_{2}\right\rangle=\frac{1}{\sqrt{2(1-|\delta|)}}\left(\left|\psi_{1}\right\rangle-e^{i \Delta t \Sigma \omega}\left|\psi_{2}\right\rangle\right), \\
& \left|e_{3}\right\rangle=\frac{1}{\sqrt{c_{3}}}\left(\left|\partial_{\Delta t} e_{1}\right\rangle-\left\langle e_{1} \mid \partial_{\Delta t} e_{1}\right\rangle\left|e_{1}\right\rangle\right), \\
& \left|e_{4}\right\rangle=\frac{1}{\sqrt{c_{4}}}\left(\left|\partial_{\Delta t} e_{2}\right\rangle-\left\langle e_{1} \mid \partial_{\Delta t} e_{2}\right\rangle\left|e_{2}\right\rangle\right),
\end{aligned}
$$

where

$$
\delta=\left\langle\psi_{1} \mid \psi_{2}\right\rangle=e^{-\frac{\Delta t^{2} \sigma^{2}}{2}-\frac{\Delta \omega^{2}}{8 \sigma^{2}}-i \Delta t \Sigma \omega},
$$

and $c_{3}$ and $c_{4}$ are normalization factors. We can diagonalize the state as

$$
\begin{aligned}
\rho & =p_{1}\left|e_{1}\right\rangle\left\langle e_{1}\left|+p_{2}\right| e_{2}\right\rangle\left\langle e_{2}\right|, \\
p_{1} & =\frac{1}{2}(1+|\delta|), \quad p_{2}=\frac{1}{2}(1-|\delta|) .
\end{aligned}
$$

The SLDs are

$$
\begin{aligned}
L_{\Delta t} & =\left(\begin{array}{cccc}
\partial_{\Delta t} p_{1} / p_{1} & 0 & 2\left\langle\partial_{\Delta t} e_{1} \mid e_{3}\right\rangle & 0 \\
0 & \partial_{\Delta t} p_{2} / p_{2} & 0 & 2\left\langle\partial_{\Delta t} e_{2} \mid e_{4}\right\rangle \\
2\left\langle e_{3} \mid \partial_{\Delta t} e_{1}\right\rangle & 0 & 0 & 0 \\
0 & 2\left\langle e_{4} \mid \partial_{\Delta t} e_{2}\right\rangle & 0 & 0
\end{array}\right), \\
L_{\Delta \omega} & =\left(\begin{array}{cccc}
\partial_{\Delta \omega} p_{1} / p_{1} & 0 & 2 i\left\langle\partial_{\Delta \omega} e_{1} \mid e_{3}\right\rangle & 0 \\
0 & \partial_{\Delta \omega} p_{2} / p_{2} & 0 & 2 i\left\langle\partial_{\Delta \omega} e_{2} \mid e_{4}\right\rangle \\
-2 i\left\langle e_{3} \mid \partial_{\Delta \omega} e_{1}\right\rangle & 0 & 0 & 0 \\
0 & -2 i\left\langle e_{4} \mid \partial_{\Delta \omega} e_{2}\right\rangle & 0 & 0
\end{array}\right),
\end{aligned}
$$


where

$$
\begin{aligned}
& \left\langle\partial_{\Delta t} e_{1} \mid e_{3}\right\rangle=\left\langle\partial_{\Delta \omega} e_{1} \mid e_{3}\right\rangle
\end{aligned}
$$

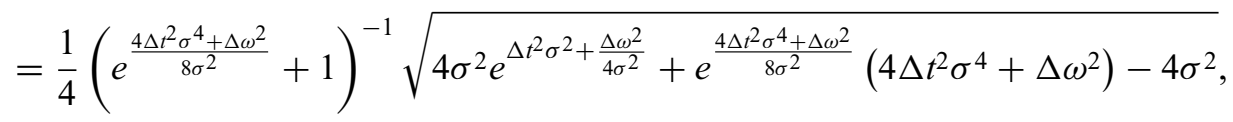

$$
\begin{aligned}
& \left\langle\partial_{\Delta t} e_{2} \mid e_{4}\right\rangle=\left\langle\partial_{\Delta \omega} e_{2} \mid e_{4}\right\rangle \\
& =\frac{1}{4}\left(e^{\frac{4 \Delta t^{2} \sigma^{4}+\Delta \omega^{2}}{8 \sigma^{2}}}-1\right)^{-1} \sqrt{4 \sigma^{2} e^{\Delta t^{2} \sigma^{2}+\frac{\Delta \omega^{2}}{4 \sigma^{2}}}-e^{\frac{4 \Delta t^{2} \sigma^{4}+\Delta \omega^{2}}{8 \sigma^{2}}}\left(4 \Delta t^{2} \sigma^{4}+\Delta \omega^{2}\right)-4 \sigma^{2} .}
\end{aligned}
$$

We finally arrive at the QFI matrix:

$$
H=\left(\begin{array}{cc}
H_{\Delta t^{2}} & H_{\Delta t \Delta \omega} \\
H_{\Delta t \Delta \omega} & H_{\Delta \omega^{2}}
\end{array}\right)
$$

where

$$
\begin{aligned}
H_{\Delta t^{2}} & =\frac{1}{4 \sigma^{2}}-\frac{\Delta \omega^{2}}{4}\left(e^{\Delta t^{2} \sigma^{2}+\frac{\Delta \omega^{2}}{4 \sigma^{2}}}-1\right)^{-1} \\
H_{\Delta \omega^{2}} & =\sigma^{2}-\frac{\Delta t^{2}}{4}\left(e^{\Delta t^{2} \sigma^{2}+\frac{\Delta \omega^{2}}{4 \sigma^{2}}}-1\right)^{-1} \\
H_{\Delta t \Delta \omega} & =\frac{\Delta t \Delta \omega}{4}\left(e^{\Delta t^{2} \sigma^{2}+\frac{\Delta \omega^{2}}{4 \sigma^{2}}}-1\right)^{-1}
\end{aligned}
$$

\section{APPENDIX E: RELATIVE RANGE AND VELOCITY ESTIMATION WITH ENTANGLED PHOTONS}

We want to compare the single photon with entangled photon pairs (similar to quantum illumination [7-9]). For a fair comparison, we send out one photon from an entangled photon pair, which has the same bandwidth. An entangled photon pair can be generated from a spontaneous parametric down conversion (SPDC) source.

If the signal photon is back-scattered, the two-photon state is described as

$$
\rho_{\mathrm{SPDC}}=\frac{1}{2}\left(\left|\Psi_{1}\right\rangle\left\langle\Psi_{1}|+| \Psi_{2}\right\rangle\left\langle\Psi_{2}\right|\right),
$$

where, in the time domain, the two-photon states are

$$
\begin{aligned}
& \left|\Psi_{1}\right\rangle=\int d t \int d t_{i} \Psi_{1}\left(t, t_{i}\right)|t\rangle\left|t_{i}\right\rangle, \\
& \left|\Psi_{2}\right\rangle=\int d t \int d t_{i} \Psi_{2}\left(t, t_{i}\right)|t\rangle\left|t_{i}\right\rangle
\end{aligned}
$$

and, for $j=1,2$,

$$
\begin{aligned}
\Psi_{j}\left(t, t_{i}\right)= & \sqrt{\frac{2}{\pi}} \sqrt[4]{1-\kappa^{2}} \sqrt{\sigma \sigma_{i}} e^{-i \bar{\omega}_{j}\left(t-\bar{t}_{j}\right)-i \bar{\omega}_{i}\left(t_{i}-\bar{t}_{i}\right)} \\
& \times \exp \left[-\left(t-\bar{t}_{j}\right)^{2} \sigma^{2}-\left(t_{i}-\bar{t}_{i}\right)^{2} \sigma_{i}^{2}+2 \kappa(t-\bar{t})\left(t_{i}-\bar{t}_{i}\right) \sigma \sigma_{i}\right] .
\end{aligned}
$$

The Hilbert space is spanned by the vectors $\left|E_{j}\right\rangle, j=1,2, \ldots, 6$.

$$
\begin{aligned}
& \left|E_{1}\right\rangle=\frac{1}{\sqrt{2\left(1+\left|\delta^{\prime}\right|\right)}}\left(\left|\Psi_{1}\right\rangle+e^{i \Delta t \Sigma \omega}\left|\Psi_{2}\right\rangle\right), \\
& \left|E_{2}\right\rangle=\frac{1}{\sqrt{2\left(1-\left|\delta^{\prime}\right|\right)}}\left(\left|\Psi_{1}\right\rangle-e^{i \Delta t \Sigma \omega}\left|\Psi_{2}\right\rangle\right),
\end{aligned}
$$




$$
\begin{aligned}
\left|E_{3}\right\rangle & =\frac{1}{\sqrt{c_{3}}}\left(\left|\partial_{\Delta t} E_{1}\right\rangle-\left\langle E_{1} \mid \partial_{\Delta t} E_{1}\right\rangle\left|E_{1}\right\rangle\right), \\
\left|E_{4}\right\rangle & =\frac{1}{\sqrt{c_{4}}}\left(\left|\partial_{\Delta t} E_{2}\right\rangle-\left\langle E_{2} \mid \partial_{\Delta t} E_{2}\right\rangle\left|E_{2}\right\rangle\right) . \\
\left|E_{5}\right\rangle & =\frac{1}{\sqrt{c_{5}}}\left(\left|\partial_{\Delta \omega} E_{1}\right\rangle-\left\langle E_{1} \mid \partial_{\Delta \omega} E_{1}\right\rangle\left|E_{1}\right\rangle-\left\langle E_{3} \mid \partial_{\Delta \omega} E_{1}\right\rangle\left|E_{3}\right\rangle\right), \\
\left|E_{6}\right\rangle & =\frac{1}{\sqrt{c_{6}}}\left(\left|\partial_{\Delta \omega} E_{2}\right\rangle-\left\langle E_{2} \mid \partial_{\Delta \omega} E_{2}\right\rangle\left|E_{2}\right\rangle-\left\langle E_{4} \mid \partial_{\Delta \omega} E_{2}\right\rangle\left|E_{4}\right\rangle\right) .
\end{aligned}
$$

The constants are

$$
\begin{aligned}
& c_{3}=\left\langle\partial_{\Delta t} E_{1} \mid \partial_{\Delta t} E_{1}\right\rangle-\left|\left\langle\partial_{\Delta t} E_{1} \mid E_{1}\right\rangle\right|^{2}, \\
& c_{4}=\left\langle\partial_{\Delta t} E_{2} \mid \partial_{\Delta t} E_{2}\right\rangle-\left|\left\langle\partial_{\Delta t} E_{2} \mid E_{2}\right\rangle\right|^{2}, \\
& c_{5}=\left\langle\partial_{\Delta \omega} E_{1} \mid \partial_{\Delta \omega} E_{1}\right\rangle-\left|\left\langle E_{1} \mid \partial_{\Delta \omega} E_{1}\right\rangle\right|^{2}-\left|\left\langle E_{3} \mid \partial_{\Delta \omega} E_{1}\right\rangle\right|^{2}, \\
& c_{6}=\left\langle\partial_{\Delta \omega} E_{2} \mid \partial_{\Delta \omega} E_{2}\right\rangle-\left|\left\langle E_{2} \mid \partial_{\Delta \omega} E_{2}\right\rangle\right|^{2}-\left|\left\langle E_{4} \mid \partial_{\Delta \omega} E_{2}\right\rangle\right|^{2} .
\end{aligned}
$$

The state can be diagonalized as

$$
\begin{aligned}
\rho_{\mathrm{SPDC}} & =P_{1}\left|E_{1}\right\rangle\left\langle E_{1}\left|+P_{2}\right| E_{2}\right\rangle\left\langle E_{2}\right|, \\
P_{1} & =\frac{1}{2}\left(1+\left|\delta^{\prime}\right|\right), \quad P_{2}=\frac{1}{2}\left(1-\left|\delta^{\prime}\right|\right), \\
\delta^{\prime} & =\left\langle\Psi_{1} \mid \Psi_{2}\right\rangle=e^{-\frac{1}{2} \Delta t^{2} \sigma_{s}^{2}-\frac{\Delta \omega^{2}}{8\left(1-\kappa^{2}\right) \sigma_{s}^{2}}-i \Delta t \Sigma \omega} .
\end{aligned}
$$

The SLDs for $\Delta t$ and $\Delta \omega$ are

$$
\begin{aligned}
& L_{\Delta t}=\left(\begin{array}{cccccc}
\partial_{\Delta t} P_{1} / P_{1} & 0 & 2\left\langle\partial_{\Delta t} E_{1} \mid E_{3}\right\rangle & 0 & 0 & 0 \\
0 & \partial_{\Delta t} p_{2} / p_{2} & 0 & \left\langle\partial_{\Delta t} E_{2} \mid E_{4}\right\rangle & 0 & 0 \\
2\left\langle E_{3} \mid \partial_{\Delta t} E_{1}\right\rangle & 0 & 0 & 0 & 0 & 0 \\
0 & \left\langle E_{4} \mid \partial_{\Delta t} E_{2}\right\rangle & 0 & 0 & 0 & 0 \\
0 & 0 & 0 & 0 & 0 & 0 \\
0 & 0 & 0 & 0 & 0 & 0
\end{array}\right) \\
& L_{\Delta \omega}=\left(\begin{array}{cccccc}
\partial_{\Delta \omega} P_{1} / P_{1} & 0 & 2\left\langle\partial_{\Delta \omega} E_{1} \mid E_{3}\right\rangle & 0 & 2\left\langle\partial_{\Delta \omega} E_{1} \mid E_{5}\right\rangle & 0 \\
0 & \partial_{\Delta \omega} P_{2} / P_{2} & 0 & 2\left\langle\partial_{\Delta \omega} E_{2} \mid E_{4}\right\rangle & 0 & 2\left\langle\partial_{\Delta \omega} E_{2} \mid E_{6}\right\rangle \\
2\left\langle E_{3} \mid \partial_{\Delta \omega} E_{1}\right\rangle & 0 & 0 & 0 & 0 & 0 \\
0 & 2\left\langle E_{4} \mid \partial_{\Delta \omega} E_{2}\right\rangle & 0 & 0 & 0 & 0 \\
2\left\langle E_{5} \mid \partial_{\Delta \omega} E_{1}\right\rangle & 0 & 0 & 0 & 0 & 0 \\
0 & 2\left\langle E_{6} \mid \partial_{\Delta \omega} E_{2}\right\rangle & 0 & 0 & 0 & 0
\end{array}\right) .
\end{aligned}
$$

The terms in the SLDs are, for example,

$$
\begin{aligned}
\left\langle\partial_{\Delta \omega} E_{1} \mid E_{3}\right\rangle & =\frac{1}{\sqrt{c_{3}}}\left(\left\langle\partial_{\Delta \omega} E_{1} \mid \partial_{\Delta t} E_{1}\right\rangle-\left\langle E_{1} \mid \partial_{\Delta t} E_{1}\right\rangle\left\langle\partial_{\Delta \omega} E_{1} \mid E_{1}\right\rangle\right), \\
\left\langle\partial_{\Delta \omega} E_{1} \mid E_{5}\right\rangle & =\frac{1}{\sqrt{c_{5}}}\left(\left\langle\partial_{\Delta \omega} E_{1} \mid \partial_{\Delta \omega} E_{1}\right\rangle-\left\langle E_{1} \mid \partial_{\Delta \omega} E_{1}\right\rangle\left\langle\partial_{\Delta \omega} E_{1} \mid E_{1}\right\rangle-\left\langle E_{3} \mid \partial_{\Delta \omega} E_{1}\right\rangle\left\langle\partial_{\Delta \omega} E_{1} \mid E_{3}\right\rangle\right) \\
& =\sqrt{c_{5}} .
\end{aligned}
$$


After some algebra, we obtain the following expression for the QFI matrix:

$$
H=\left(\begin{array}{cc}
Q_{\Delta t^{2}} & Q_{\Delta t \Delta \omega} \\
Q_{\Delta t \Delta \omega} & Q_{\Delta \omega^{2}}
\end{array}\right)
$$

with

$$
\begin{aligned}
Q_{\Delta t^{2}} & =\sigma^{2}-\frac{\Delta \omega^{2}}{4}\left(e^{\Delta t^{2} \sigma^{2}+\frac{\Delta \omega^{2}}{4\left(1-\kappa^{2}\right) \sigma^{2}}}-1\right)^{-1}, \\
Q_{\Delta \omega^{2}} & =\frac{1}{1-\kappa^{2}} \frac{1}{4 \sigma^{2}}-\frac{\Delta t^{2}}{4}\left(e^{\Delta t^{2} \sigma^{2}+\frac{\Delta \omega^{2}}{4\left(1-\kappa^{2}\right) \sigma^{2}}}-1\right)^{-1}, \\
Q_{\Delta t \Delta \omega} & =\frac{\Delta t \Delta \omega}{4}\left(e^{\Delta t^{2} \sigma^{2}+\frac{\Delta \omega^{2}}{4\left(1-\kappa^{2}\right) \sigma^{2}}}-1\right)^{-1} .
\end{aligned}
$$

The expectation value of the commutation relation then becomes

$$
\operatorname{Tr}\left(\rho\left[L_{\Delta t}, L_{\Delta \omega}\right]\right)=i\left(e^{\Delta t^{2} \sigma^{2}+\frac{\Delta \omega^{2}}{4\left(1-\kappa^{2}\right) \sigma^{2}}}-1\right)^{-1}\left(\Delta t^{2} \sigma^{2}+\frac{\Delta \omega^{2}}{4\left(1-\kappa^{2}\right) \sigma^{2}}\right)-i
$$

\section{APPENDIX F: A SIMULTANEOUS MEASUREMENT OF TIME AND FREQUENCY}

In this appendix, we present the construction by Zhuang et al. [26] for the optimal measurement of time and frequency estimation. We consider a simplified model where the bandwidth of the signal photon remains constant. Consider the two-photon state given, in the time domain, by Eq. (27), where we put $\sigma=\sigma_{i 0}$. To simplify the notation, we put $\bar{t}_{i 0}=0$ and $\bar{\omega}_{i 0}=0$. The state factorizes when written in terms of the variables $t_{+}:=t+t_{i}$ and $t_{-}:=t-t_{i}$ :

$$
\Psi\left(t_{+}, t_{-}\right) \sim \exp \left[-\frac{\left(t_{+}-\bar{t}\right)^{2}(1-\kappa) \sigma^{2}}{2}-\frac{i \bar{\omega}\left(t_{+}-\bar{t}\right)}{2}\right] \exp \left[-\frac{\left(t_{-}-\bar{t}\right)^{2}(1+\kappa) \sigma^{2}}{2}-\frac{i \bar{\omega}\left(t_{-}-\bar{t}\right)}{2}\right]
$$

We can Fourier transform the variable $t_{+}$and express the state in terms of $\omega_{+}=\omega+\omega_{i}$ and $t_{-}$:

$$
\tilde{\Psi}\left(\omega_{+}, \tau_{-}\right) \sim \exp \left[-\frac{\left(2 \omega_{+}-\bar{\omega}\right)^{2}}{8(1-\kappa) \sigma^{2}}+i \omega_{+} \bar{t}\right] \exp \left[-\frac{\left(t_{-}-\bar{t}\right)^{2}(1+\kappa) \sigma^{2}}{2}-\frac{i \bar{\omega}\left(t_{-}-\bar{t}\right)}{2}\right] .
$$

This shows that we can jointly estimate $\bar{\omega}$ and $\bar{t}$ by first splitting the two photons and then by applying intensity measurements in the variable $\omega_{+}$and $t_{-}$. The probability density of measuring a photon at frequency $\omega_{+}$and the other photon at time $t_{-}$is

$P\left(\omega_{+}, t_{-}\right) \sim \exp \left[-\frac{\left(2 \omega_{+}-\bar{\omega}\right)^{2}}{4(1-\kappa) \sigma^{2}}\right] \exp \left[-\left(t_{-}-\bar{t}\right)^{2}(1+\kappa) \sigma^{2}\right]$

It follows that $\bar{\omega}$ and $\bar{t}$ can be estimated in this way with mean-square errors

$$
\delta t^{2}=\frac{1}{2(1+\kappa) \sigma^{2}}, \quad \delta \omega^{2}=2(1-\kappa) \sigma^{2}
$$

Putting this into the QCR bound, we obtain

$$
\begin{gathered}
\delta t^{2}=\frac{1}{2(1+\kappa) \sigma^{2}} \geqslant \frac{1}{4 \sigma^{2}}, \\
\delta \omega^{2}=2(1-\kappa) \sigma^{2} \geqslant\left(1-\kappa^{2}\right) \sigma^{2} .
\end{gathered}
$$

In conclusion, this shows that this joint measurement is almost optimal for $\kappa \simeq 1$ and saturates the QCR bound in the limit that $\kappa \rightarrow 1$, i.e., perfect time-frequency correlations.

\section{APPENDIX G: UNEVEN SOURCE BRIGHTNESS}

In this appendix, we consider the effect of unequal brightness of the two sources, assuming $\Delta \omega \rightarrow 0$. For the 
state

$$
\rho=q\left|\psi_{1}\right\rangle\left\langle\psi_{1}|+(1-q)| \psi_{2}\right\rangle\left\langle\psi_{2}\right|
$$

one can relax the condition that $q=1 / 2$. The problem of having uneven strength of the sources has been considered by Řehaček et al. in Ref. [45]. There are two scenarios to consider:

(1) The case where the centroid $\bar{t}_{0}$ is known.

(2) The case where all three parameters $\left(\bar{t}_{0}, \Delta t, q\right)$ need to be estimated.

The QFI matrix for the three parameters $\left(\bar{t}_{0}, \Delta t, q\right)$ becomes [45]

$$
\begin{aligned}
& Q\left(\bar{t}_{0}, \Delta t, q\right)=4 \\
& \quad \times\left(\begin{array}{ccc}
\sigma^{2}+4 q(1-q) P & (q-1 / 2) \sigma^{2} & -i \delta P \\
(q-1 / 2) \sigma^{2} & \sigma^{2} / 4 & 0 \\
-i \delta P & 0 & \frac{1-w^{2}}{4 q(1-q)}
\end{array}\right) \\
& \delta=\exp \left(-\Delta t^{2} \sigma^{2} / 2\right) \\
& P=i \Delta t \sigma^{2} \exp \left(-\frac{\Delta t^{2} \sigma^{2}}{2}\right)
\end{aligned}
$$

For case 1, we note that the element $Q_{\Delta t}=\sigma^{2}$ and is independent of $q$. This means that if $\bar{t}_{0}$ and $q$ are known, then the results in Fig. 4 are achievable, despite $q \neq 1 / 2$. For case 2, we need to take into account the multiparameter estimation problem of estimating the centroid, the separation and the relative strengths of the sources.

We plot the QFI for different values of $q$ in Fig. 6 and the corresponding FI for direct imaging in the same color. Assuming that we do not know the centroid precisely, we see that in order to have a constant QFI, we require

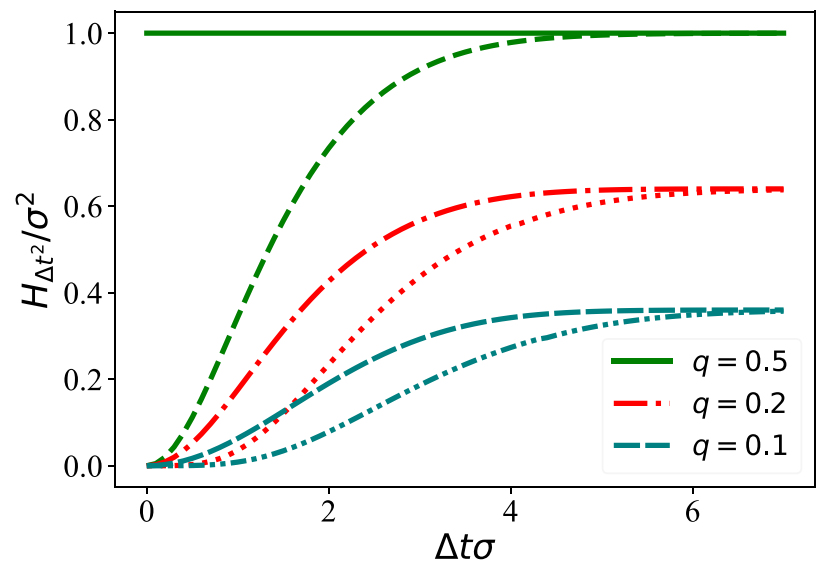

FIG. 6. The QFI and the FI for different reflectivities of the sources. Each color is a different $q$ and the QFI is always larger than the FI from direct measurement. $q=0.5$. Despite this, when $q \neq 0.5$, one can still always resolve the separation parameter better than when using the direct intensity measurement, i.e., the QFI is higher for all parameters of the separation.

[1] V. Giovannetti, S. Lloyd, and L. Maccone, Quantumenhanced measurements: Beating the standard quantum limit, Science 306, 1330 (2004).

[2] V. Giovannetti, S. Lloyd, and L. Maccone, Quantum Metrology, Phys. Rev. Lett. 96, 010401 (2006).

[3] M. I. Kolobov, Quantum Imaging (Springer Science \& Business Media, New York, 2007).

[4] J. D. Franson, Bell Inequality for Position and Time, Phys. Rev. Lett. 62, 2205 (1989).

[5] M. D'Angelo, M. V. Chekhova, and Y. Shih, Two-Photon Diffraction and Quantum Lithography, Phys. Rev. Lett. 87, 013602 (2001).

[6] V. Giovannetti, S. Lloyd, and L. Maccone, Quantumenhanced positioning and clock synchronization, Nature 412, 417 (2001).

[7] S. Lloyd, Enhanced sensitivity of photodetection via quantum Illumination, Science 321, 1463 (2008).

[8] S.-H. Tan, B. I. Erkmen, V. Giovannetti, S. Guha, S. Lloyd, L. Maccone, S. Pirandola, and J. H. Shapiro, Quantum Illumination with Gaussian States, Phys. Rev. Lett. 101, 253601 (2008).

[9] S. Barzanjeh, S. Guha, C. Weedbrook, D. Vitali, J. H. Shapiro, and S. Pirandola, Microwave Quantum Illumination, Phys. Rev. Lett. 114, 080503 (2015).

[10] Z. Huang, C. Macchiavello, and L. Maccone, Usefulness of entanglement-assisted quantum metrology, Phys. Rev. A 94, 012101 (2016).

[11] Z. Huang, C. Macchiavello, L. Maccone, and P. Kok, Ancilla-assisted schemes are beneficial for Gaussian state phase estimation, Phys. Rev. A 101, 012124 (2020).

[12] Z. Huang, C. Macchiavello, and L. Maccone, Noisedependent optimal strategies for quantum metrology, Phys. Rev. A 97, 032333 (2018).

[13] J. H. Shapiro and R. W. Boyd, The physics of ghost imaging, Quantum Inf. Process. 11, 949 (2012).

[14] A. H. Zewail, Femtochemistry: Atomic-scale dynamics of the chemical bond, J. Phys. Chem. A 104, 5660 (2000).

[15] A. McCarthy, R. J. Collins, N. J. Krichel, V. Fernández, A. M. Wallace, and G. S. Buller, Long-range time-offlight scanning sensor based on high-speed time-correlated single-photon counting, Appl. Opt. 48, 6241 (2009).

[16] A. McCarthy, N. J. Krichel, N. R. Gemmell, X. Ren, M. G. Tanner, S. N. Dorenbos, V. Zwiller, R. H. Hadfield, and G. S. Buller, Kilometer-range, high resolution depth imaging via $1560 \mathrm{~nm}$ wavelength single-photon detection, Opt. Express 21, 8904 (2013).

[17] S. T. Cundiff and J. Ye, Colloquium: Femtosecond optical frequency combs, Rev. Mod. Phys. 75, 325 (2003).

[18] H. Liu and A. S. Helmy, IBM Q experience as a versatile experimental testbed for simulating open quantum systems, Npj Quantum Inf. 6, 1 (2020). 
[19] J. Nunn, L. Wright, C. Söller, L. Zhang, I. Walmsley, and B. Smith, Large-alphabet time-frequency entangled quantum key distribution by means of time-to-frequency conversion, Opt. Express 21, 15959 (2013).

[20] Z. Zhang, J. Mower, D. Englund, F. N. C. Wong, and J. H. Shapiro, Unconditional Security of Time-Energy Entanglement Quantum Key Distribution Using Dual-Basis Interferometry, Phys. Rev. Lett. 112, 120506 (2014).

[21] M. I. Skolnik, IRE Transactions on Aeronautical and Navigational Electronics, 123 (1960).

[22] G. R. Curry, Radar System Performance Modeling (Artech House, Norwood, Massachusetts, 2005).

[23] E. Arthurs and J. Kelly, On the simultaneous measurement of a pair of conjugate observables, Bell Syst. Tech. J. 44, 725 (1965).

[24] J.-P. W. MacLean, J. M. Donohue, and K. J. Resch, Direct Characterization of Ultrafast Energy-Time Entangled Photon Pairs, Phys. Rev. Lett. 120, 053601 (2018).

[25] M. Berta, M. Christandl, R. Colbeck, J. M. Renes, and R. Renner, The uncertainty principle in the presence of quantum memory, Nature Phys. 6, 659 (2010).

[26] Q. Zhuang, Z. Zhang, and J. H. Shapiro, Entanglementenhanced lidars for simultaneous range and velocity measurements, Phys. Rev. A 96, 040304 (2017).

[27] J. M. Donohue, V. Ansari, J. Řeháček, Z. Hradil, B. Stoklasa, M. Paúr, L. L. Sánchez-Soto, and C. Silberhorn, Quantum-Limited Time-Frequency Estimation through Mode-Selective Photon Measurement, Phys. Rev. Lett. 121, 090501 (2018).

[28] V. Ansari, B. Brecht, J. Gil-López, J. M. Donohue, J. Řeháček, Z. Hradil, L. L. Sánchez-Soto, and C. Silberhorn, arXiv:2009.01069 (2020).

[29] S. L. Braunstein and C. M. Caves, Statistical Distance and the Geometry of Quantum States, Phys. Rev. Lett. 72, 3439 (1994).

[30] I. Afnan, R. Banerjee, S. L. Braunstein, I. Brevik, C. M. Caves, B. Chakraborty, E. Fischbach, L. Lindblom, G. Milburn, and S. Odintsov et al., Author index for volume 247, Ann. Phys. 247, 447 (1996).

[31] M. G. Paris, Quantum estimation for quantum technology, Int. J. Quantum Inf. 7, 125 (2009).

[32] M. Jarzyna and R. Demkowicz-Dobrzański, Matrix Product States for Quantum Metrology, Phys. Rev. Lett. 110, 240405 (2013).
[33] S. Ragy, M. Jarzyna, and R. Demkowicz-Dobrzański, Compatibility in multiparameter quantum metrology, Phys. Rev. A 94, 052108 (2016).

[34] S. M. Kay, Fundamentals of Statistical Signal Processing (Prentice Hall PTR, Englewood Cliffs, NJ, 1993).

[35] Note that, in general, the attainability requires a collective measurement on multiple copies for mixed states (so $N$ is not simply the number of repetitions of the experiment). On the other hand, if the state is pure, then separable measurements on each copy are sufficient [46].

[36] A. Fujiwara, Multi-parameter pure state estimation based on the right logarithmic derivative, Math. Eng. Tech. Rep. 94.9, (1994).

[37] There is no general hierarchy between RLD and SLD bounds $[46,47]$.

[38] J.-C. Diels and W. Rudolph, Ultrashort Laser Pulse Phenomena (Elsevier, New York, 2006).

[39] M. Tsang, R. Nair, and X.-M. Lu, Quantum Theory of Superresolution for Two Incoherent Optical Point Sources, Phys. Rev. X 6, 031033 (2016).

[40] R. Komissarov, V. Kozlov, D. Filonov, and P. Ginzburg, Partially coherent radar unties range resolution from bandwidth limitations, Nat. Commun. 10, 1 (2019).

[41] C. Lupo, Z. Huang, and P. Kok, Quantum Limits to Incoherent Imaging Are Achieved by Linear Interferometry, Phys. Rev. Lett. 124, 080503 (2020).

[42] We relax this condition and consider having uneven reflectivities for the unentangled case in Appendix G.

[43] B. Roy Frieden, Science from Fisher information: a unification (Cambridge University Press, Cambridge, 2004).

[44] J. M. Lukens and P. Lougovski, Frequency-encoded photonic qubits for scalable quantum information processing, Optica 4, 8 (2017).

[45] J. Řehaček, Z. Hradil, B. Stoklasa, M. Paúr, J. Grover, A. Krzic, and L. L. Sánchez-Soto, Multiparameter quantum metrology of incoherent point sources: Towards realistic superresolution, Phys. Rev. A 96, 062107 (2017).

[46] F. Albarelli, M. Barbieri, M. G. Genoni, and I. Gianani, A perspective on multiparameter quantum metrology: From theoretical tools to applications in quantum imaging, Phys. Lett. A 384, 126311 (2020).

[47] M. Bradshaw, P. K. Lam, and S. M. Assad, Ultimate precision of joint quadrature parameter estimation with a Gaussian probe, Phys. Rev. A 97, 012106 (2018). 